DIVISION OF THE HUMANITIES AND SOCIAL SCIENCES

CALIFORNIA INSTITUTE OF TECHNOLOGY

PASADENA, CALIFORNIA 91125

THE FORMATION OF NETWORKS WITH TRANSFERS AMONG PLAYERS

Francis Bloch

GREQAM, Université d'Aix-Marseille

Matthew O. Jackson

California Institute of Technology

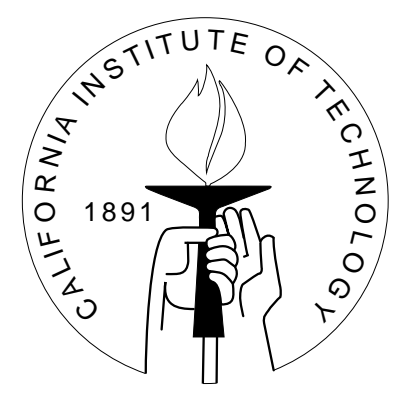

SOCIAL SCIENCE WORKING PAPER 1194

April 2004 


\title{
The Formation of Networks with Transfers among Players
}

\author{
Francis Bloch Matthew O. Jackson
}

\begin{abstract}
We examine the formation of networks among a set of players whose payoffs depend on the structure of the network. We focus on games where players may bargain by promising or demanding transfer payments when forming links. We examine several variations of the transfer/bargaining aspect of link formation. One aspect is whether players can only make and receive transfers to other players to whom they are directly linked, or whether they can also subsidize links that they are not directly involved in. Another aspect is whether or not transfers related to a given link can be made contingent on the full resulting network or only on the link itself. A final aspect is whether or not players can pay other players to refrain from forming links. We characterize the networks that are supported under these variations and show how each of the above aspects is related either to accounting for a specific type of externality, or to dealing with the combinatorial nature of network payoffs.
\end{abstract}

JEL classification numbers: A14, C71, C72

Key words: Networks, Network Games, Network Formation, Game Theory, Efficient Networks, Side Payments, Transfers, Bargaining, Externalities 


\title{
The Formation of Networks with Transfers among Players*
}

\author{
Francis Bloch Matthew O. Jackson
}

\section{Introduction}

Many social, economic, and political interactions take the form of a network of bilateral relationships. This ranges from friendships to trading relationships and political alliances. As the structure of the network of relationships can have a profound impact on the welfare of all the involved parties, it is essential to develop a good understanding of which networks are likely to form and how this depends on the specifics of the circumstances. This paper contributes to a growing literature that models network formation. ${ }^{1}$

Here, our focus is on the role played by transfers payments in the formation of social and economic networks. In many applications, agents bargain on possible transfers at the time of forming relationships. For example, when two airlines form a code-sharing agreement, included in that agreement are the details of how the costs and revenues on cross-booked passengers are to be split. Similarly, when two political parties form an electoral pact, they explicitly or implicitly agree on the division of seats, committee positions, cabinet posts, and government benefits. Without transfer payments (in currency or in kind), many agreements would simply never exist.

Our first objective in this paper is to construct a simple model where the agreement on transfers is part of the process of the formation of links. Our second objective is to study how the formation of networks depends on the types of transfers that agents can make. How important is it that agents can subsidize the formation of links that they are not directly involved in? How important is it that agents be able to make payments contingent on the full network that emerges? What is the role of making payments to other players if they refrain from forming links? Since the types of payments that agents will have at their discretion depends on the application, the answers to these questions

${ }^{*}$ Financial support from the Lee Center for Advanced Networking and from the NSF under grant SES-0316493 is gratefully acknowledged. We thank Anke Gerber and the participants of the Ninth Coalition Theory Network Workshop for a helpful discussion of the paper, and Toni Calvo-Armengol for comments on an earlier draft.

${ }^{1}$ See Jackson (2003b) for a survey of the literature that is most closely related to our work here. 
help us to understand the relationship between the networks that emerge, and for instance whether efficient networks form, and the specifics of the social or economic interaction.

Our results outline some simple and intuitive relationships between the types of transfers available and the networks that emerge. The main results can be summarized as follows. If transfers can only be made between the players directly involved in a link, then the set of networks that emerge as equilibria are characterized by a balance condition. While there are some settings where efficient networks are supported with only direct transfers, there are many settings where the networks that form will be inefficient. If players can make indirect transfers, so that they can subsidize the formation of links between other players, then they can properly account for some forms of positive externalities. However, even with indirect transfers, we still need to worry about the fact that there are many different combinations of links that players might consider forming or not forming. Thus, even though links are bilateral, the multitude of such relationships results in some multilateral decision problems. This means that in order to guarantee that efficient networks form, players need not only to be able to make indirect transfers in order to deal with (positive) externalities, but also to make those transfers contingent on the network that emerges in order to take care of the multitude of interrelated bilateral problems. Thus, there is a basic sense in which one can view the role of indirect payments as taking care of externalities, and contingencies as taking care of the combinatorial nature of network formation. Finally, in order to handle negative externalities, players need to be able to pay other players not to form links. Our analysis also includes some discussion of how to model equilibrium, and we defer all discussion of that analysis until we have laid out the details of the network formation games. This outlining of the relationship between the types of transfers admitted and the types of externalities and the multilateral decision problem that are overcome is the first that we know of in the networks literature, or even the contracting literature for that matter.

Before presenting the model, let us briefly discuss its relationship to the most closely related literature. This paper fits into a recent literature that examines network formation when players act in their own interest and their payoffs may depend on the whole structure of the network. ${ }^{2}$ In such network games, Jackson and Wolinsky (1996) showed that the networks that maximize society's overall payoff will often not be stable in an equilibrium sense, regardless of how players' payoffs are allocated or re-allocated (subject to two basic conditions of anonymity and component balancedness). ${ }^{3}$ Moreover, simple examples showed that even when players have the ability to make side-payments, efficient networks may fail to form because side-payments do not enable players to overcome the difficulties linked with network externalities.

This tension between efficiency and stability underlies our analysis of link formation with transfers, and we develop a deeper understanding of the source of such inefficiencies.

\footnotetext{
${ }^{2}$ See Jackson (2003a) for a survey of this literature; as well as Slikker and van den Nouweland (2001a) for a look at the literature that deals with communication structures in cooperative game theory, where a graph structure determines which coalitions can generate value.

${ }^{3}$ See Jackson and Wolinsky (1996) and Dutta and Mutuswami (1997) for detailed discussion of the role of the conditions.
} 
We identify two reasons why side-payments may be ineffective in resolving the conflict between efficiency and stability. First, there is the fairly obvious point that widespread externalities in the network may imply that agents have to have input into the formation of links by other players for the efficient network to form. For example, if the efficient network involves the formation of a link between two players who get a negative utility from that link, side-payments will be ineffective in reaching the efficient outcome. Second, is the less obvious point that since players are involved in multiple bilateral relationships at the same time, side-payments negotiated bilaterally may not be sufficient to sustain the formation of efficient networks. In some situations, players may have an incentive to renege on different relationships at once, even though each bilateral relationship can be sustained by side-payments. The main message of this paper is that the two difficulties identified above can be overcome by enlarging the range of possible transfers, and can be traced to specific features of the transfers. Network externalities can be dealt with if players have the ability to make indirect transfers, subsidizing the formation of links by other players or paying players not to form links. The combinatorial difficulties linked to the multitude of bilateral relationships can be solved if players have the ability to make contingent transfers depending on the network being formed. In particular, if players can make indirect contingent transfers, efficient networks can be sustained by individual incentives under very mild regularity conditions.

Ours is not the first paper to look at the endogenous determination of payoffs together with network formation. Recent models of network formation by Currarini and Morelli (2000) and Mutuswami and Winter $(2002)^{4}$ allow players to simultaneously bargain over the formation of links and the allocation of value. In particular, Currarini and Morelli (2000), and Mutuswami and Winter (2002), model network formation as a sequential process where players move in turn and announce the total payoff that they demand from the eventual network that will emerge, as well as the specific links that they are willing form. The network that forms as a function of the announcements is the largest one such that the total demands are compatible with the total value that is generated. They show that the equilibria of such games are efficient networks, assuming that there are no externalities across network components and that some other payoff monotonicity conditions are satisfied. Part of the intuition is that by moving in sequence and making such take it or leave it demands, players can extract their marginal contribution to an efficient network, and this provides correct incentives in some situations.

Currarini and Morelli (2000) and Mutuswami and Winter (2002) make the important point that the ability to determine payoffs in conjunction with link formation may aid in the emergence of efficient networks. However, these sequential games have special features and are better for illustrating the importance of taking such bargaining seriously (or for implementing variations on the Shapley value), than for providing reasonable models of network formation. In particular, the end-gaming and finite extensive forms drive the results. Moreover, while they provide some sufficient conditions for the support of efficient networks, they do not give us much of a feel for how generally this might hold, or how this depends on the structure of the process. In particular, the nature of the game

\footnotetext{
${ }^{4}$ See also Slikker and van den Nouweland (2001b) in the context of communication games.
} 
does not even allow an analysis of which players pay which others - essentially everything is implicitly centralized. ${ }^{5}$

The rest of this paper is organized as follows. Section 2 introduces our notations for players and networks. We describe the different models of network formation in Section 3. We then study the different models in turn. Section 4 is devoted to the direct transfer game, Section 5 to the indirect transfer game, Section 6 to contingent transfers and Section 7 to a game where players may pay to prevent the formation of links by other players. We conclude in Section 8. The paper ends with two Appendices. Appendix A discusses the relation between pairwise stability, as defined by Jackson and Wolinsky (1996), and the networks supported by the direct transfer game. Appendix B contains the proofs of our results.

\section{Modeling Networks}

\section{Players and Networks}

$N=\{1, \ldots, n\}$ is the set of players who may be involved in a network relationship. ${ }^{6}$

A network $g$ is a list of pairs of players who are linked to each other. For simplicity, we denote the link between $i$ and $j$ by $i j$, so $i j \in g$ indicates that $i$ and $j$ are linked in the network $g$. Let $g^{N}$ be the set of all subsets of $N$ of size 2. The network $g^{N}$ is referred to as the complete network. The set $G=\left\{g \subset g^{N}\right\}$ denotes the set of all possible networks on $N$.

For any network $g \in G$, let $N(g)$ be the set of players who have at least one link in the network $g$. That is, $N(g)=\{i \mid \exists j$ s.t. $i j \in g\}$. Given a player $i \in N$ and a network $g \in G$, let $L_{i}(g)$ denote the set of links in $g$ involving player $i, L_{i}(g)=\{j k \in g \mid j=i$ or $k=i\}$.

\section{Paths and Components}

A path in a network $g \in G$ between players $i$ and $j$ is a sequence of players $i_{1}, \ldots, i_{K}$ such that $i_{k} i_{k+1} \in g$ for each $k \in\{1, \ldots, K-1\}$, with $i_{1}=i$ and $i_{K}=j$.

A component of a network $g$, is a nonempty subnetwork $g^{\prime} \subset g$, such that

- if $i \in N\left(g^{\prime}\right)$ and $j \in N\left(g^{\prime}\right)$ where $j \neq i$, then there exists a path in $g^{\prime}$ between $i$ and $j$, and

\footnotetext{
${ }^{5}$ We have become aware of independent work by Matsubayashi and Yamakawa (2004) who analyze a game which operates on a link by link basis, as do some of the games we study here. Their work focuses on Jackson and Wolinsky's (1996) connections model, and a game where players negotiate over how much of the cost of a link each player will bear. Thus, there is almost no overlap with our results.

${ }^{6}$ For background and discussion of the model of networks discussed here, see Jackson (2003b).
} 
- if $i \in N\left(g^{\prime}\right)$ and $i j \in g$, then $i j \in g^{\prime}$.

\section{Utility Functions}

The utility of a network to player $i$ is given by a function $u_{i}: G \rightarrow \mathbb{R}_{+}{ }^{7}$ Let $u$ denote the vector of functions $u=\left(u_{1}, \ldots, u_{n}\right)$. We normalize payoffs so that $u_{i}(\emptyset)=0$.

A utility function tells us what value accrues to any given player as a function of the network. This might include all sorts of costs, benefits, and externalities.

For any network $g \in G$ and subset of links $\ell \subset g$, we define the marginal utility of the links $\ell$ in $g$ to player $i$ by $m u_{i}(g, \ell)=u_{i}(g)-u_{i}(g \backslash \ell)$.

\section{Externalities}

While the class of utility functions we consider is completely general, the following definitions of externalities will prove useful.

A profile of utility functions $u$ satisfies no externalities if $u_{i}(g)=u_{i}(g+j k)$ for all $g$, $j k \notin g$, and $i \notin j k$.

A profile of utility functions $u$ satisfies nonpositive externalities if $u_{i}(g) \geq u_{i}(g+j k)$ for all $g, j k \notin g$, and $i \notin j k$.

A profile of utility functions $u$ satisfies nonnegative externalities if $u_{i}(g) \leq u_{i}(g+j k)$ for all $g, j k \notin g$, and $i \notin j k$.

These definitions of externalities are not exhaustive since there are settings where some links may result in positive externalities and others in negative externalities, or the nature of the externality may differ across players. Nevertheless, these definitions provide a useful organizing device, and can easily be interpreted. Situations with no externalities correspond to cases where players only care about who they are connected to, but no further information. Nonpositive (negative) externalities arise when players are hurt by the formation of links by other players. An example of this is the coauthor model of Jackson and Wolinsky (1996), where a player is hurt if their co-authors take on other co-authors. Other examples of these are seen in Goyal and Joshi (2003), where two firms form strategic alliances and other firms are harmed by the resulting reduction in marginal cost; or in Goyal and Joshi (2000) and Furusawa and Konishi (2002), where two countries enter into a free-trade agreement and other countries suffer. Nonnegative (positive) externalities arise when players benefit from the formation of new links. In Jackson and Wolinsky's (1996) and connections model, externalities are

\footnotetext{
${ }^{7}$ As opposed to Jackson and Wolinsky (1996) we do not distinguish between a value function and an allocation rule. Instead, our primitive is the set of individual values for every network.
} 
positive as all players benefit from an increase in the friendship/communication network. Positive externalities also emerge in Belleflamme and Bloch (2001)'s collusive networks, where market sharing agreements reduce the number of competitors on the market to the benefit of other firms.

\section{Values and Efficiency}

A network $g \in G$ Pareto dominates a network $g^{\prime} \in G$ relative to $u$ if $u_{i}(g) \geq u_{i}\left(g^{\prime}\right)$ for all $i \in N$, with strict inequality for at least one $i \in N$. A network $g \in G$ is Pareto efficient relative to $u$ if it is not Pareto dominated.

A network $g \in G$ is efficient relative to $u$ if it maximizes $\sum_{i} u_{i}(g)$.

When transfers are possible, Pareto efficiency and efficiency are equivalent, so we focus here on efficient networks. ${ }^{8}$

\section{Network Formation Games}

We consider several models of network formation where various types of transfers are available, and examine which networks emerge as equilibria of these games. There are two basic versions of the game, allowing for direct or indirect transfers. In the direct transfer game, players can only bargain over the distribution of payoffs of the links they are involved with. In the indirect transfer game, players can subsidize the formation of links by other players. We later extend both games to allow for contingent transfers.

\section{The Direct Transfer Network Formation Game}

In the direct transfer game, every player $i \in N$ announces a vector of transfers $t^{i} \in \mathbb{R}^{n-1}$. We denote the entries in this vector by $t_{i j}^{i}$, representing the transfer that player $i$ proposes on link $i j$. Announcements are simultaneous.

Link $i j$ is formed if and only if $t_{i j}^{i}+t_{i j}^{j} \geq 0$. Formally, the network that forms as a function of the profile of announced vectors of transfers $t=\left(t^{1}, \ldots, t^{n}\right)$ is

$$
g(t)=\left\{i j \mid t_{i j}^{i}+t_{i j}^{j} \geq 0\right\}
$$

In this game, player $i$ 's payoff is given by

$$
u_{i}(g(t))-\sum_{i j \in g(t)} t_{i j}^{i}
$$

\footnotetext{
${ }^{8}$ For a detailed discussion of various notions of efficient networks in the presence of transfers, see Jackson (2003a).
} 
This game is easily interpreted. Players simultaneously announce a transfer for each possible link that they might form. If the transfer is positive, it represents the offer that the player makes to form the link. If the transfer is negative, it represents the demand that a player requests to form the link. Note that the offer may exceed the demand, $t_{i j}^{i}+t_{i j}^{j}>0$. In that case, we hold both players to their promises. If for instance $t_{i j}^{i}>-t_{i j}^{j}>0$, player $i$ ends up making a bigger payment than player $j$ demanded. Player $j$ only gets his demand, and the excess payment is wasted.

It is important to note that wasted transfers will never occur in equilibrium, and alternative specifications of the game (for instance, letting player $i$ only pay player $j$ 's demand or player $j$ receive the total offer of player $i$ ) would not change the structure of the equilibria.

\section{The Indirect Transfer Network Formation Game}

In the indirect transfer game, every player $i$ announces a vector of transfers $t^{i} \in$ $\mathbb{R}^{n(n-1) / 2}$. The entries in the vector $t^{i}$ are given by $t_{j k}^{i}$, denoting the transfer that player $i$ puts on the link $j k$. If $i \notin j k, t_{j k}^{i} \geq 0$. Player $i$ can make demands on the links that he or she involved with (it is permissible to have $t_{i j}^{i}<0$ ), but can only make offers on the other links. The reasoning here is that a player cannot prevent the formation of a link between two other players (except possibly by paying them not to form the link, as we consider later).

Link $j k$ is formed if and only if $\sum_{i \in N} t_{j k}^{i} \geq 0$. Formally, the network that forms as a function of the profile of announced vectors of potential transfers $t=\left(t^{1}, \ldots, t^{n}\right)$ is

$$
g(t)=\left\{i j \mid \sum_{i \in N} t_{j k}^{i} \geq 0\right\}
$$

In this game, player $i$ 's payoff is given by

$$
u_{i}(g(t))-\sum_{j k \in g(t)} t_{j k}^{i} .
$$

\section{Network Formation Games with Contingent Transfers}

In the games we have defined above, players only have a limited ability to condition their actions on the actions of other players. Those games do not allow for contingent contracts of the form "I will pay you to form link $i j$ only if link $j k$ is also formed." It turns out that being able to make this kind of contingent contract can be very important, and so we now define such games. 
Every player announces a vector of contingent transfers $t^{i}(g)$ contingent on $g$ forming, for each conceivable nonempty $g \in G$. In the direct transfer game, $t^{i}(g) \in \mathbb{R}^{n-1}$ for each $i$, while in the indirect transfer game, $t^{i}(g) \in \mathbb{R}^{n-1 !}$

There are many possible ways to determine which network forms given a set of contingent announcements. We consider the following one, but it will become clear that the results are robust to changes in the way the network is determined. Let there be an ordering over $G$, captured by a function $\sigma$ which maps $G$ onto $\{1, \ldots, \# G\}$. The network that forms is determined as follows. Start with the first network, $g^{1}$ such that $\sigma\left(g^{1}\right)=1$, and check whether $g\left(t\left(g^{1}\right)\right)=g^{1}$. If the answer is yes, then this is the network that forms. Otherwise, move on to the second network, $g^{2}$, and continue the process until we find such a network. The network formed is thus the first network $g^{k}$ in the ordering for which $g\left(t\left(g^{k}\right)\right)=g^{k}$. If there is no such $k$, then the empty network forms.

\section{Equilibrium and Supporting a Network}

Given a vector of transfers $t$ in any of the variants of the game, a players payoff is given by

$$
\pi_{i}(t)=u_{i}(g(t))-\sum_{j k \in g(t)} t_{j k}^{i}
$$

in the non-contingent game, ${ }^{9}$ and

$$
\pi_{i}(t)=u_{i}(g(t))-\sum_{j k \in g(t)} t_{j k}^{i}(g(t))
$$

in the contingent game.

A vector $t$ forms an equilibrium of one of the above games if it is a pure strategy Nash equilibrium of the game. That is, $t$ is an equilibrium if

$$
\pi(t) \geq \pi\left(t_{-i}, \widehat{t}^{i}\right)
$$

for all $i$ and $\widehat{t}^{\imath}$.

We say that a network $g$ is supported via a given game relative to a profile of utility functions $u=\left(u_{1}, \ldots, u_{n}\right)$ if there exists an equilibrium $t$ of the game such that $g(t)=g$.

\section{A Comment on Simultaneous Move Games}

A critical advantage of considering a simultaneous version of network formation is that after seeing the resulting network and transfers, players will not wish to make further

\footnotetext{
${ }^{9}$ This equation includes $t_{j k}^{i}$, even when $i \notin j k$, and such transfers are not included in the direct transfer game. Simply set $t_{j k}^{i}=0$ when $i \notin j k$ for the direct transfer game.
} 
changes to their transfers and links. This is not true if one instead models network formation sequentially, by having the players move in some order. It could be that the resulting network and transfers would not be stable if players could then come back and make further changes.

Regardless of whether one thinks that network formation is simultaneous, the conditions imposed by equilibrium are necessary conditions for any process to come to a stable position. That is, the equilibrium conditions that are derived here are conditions that capture the idea that we have arrived at a network such that no players would gain from further changes.

\section{A Refinement: Pairwise Equilibrium}

The simultaneity of announcements has a drawback; but one that we can easily deal with. It allows for a multiplicity of equilibrium networks as a result of coordination failures. Consider for example the following example where all the transfer games are equivalent.

Example 1 Why refine?

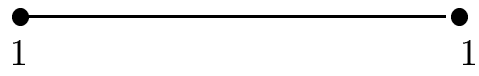

There are two supported networks. One is the empty network and the other is complete network (one link). The complete network is supported by transfers $t_{12}^{1}=t_{12}^{2}=0$. To support the empty network, set $t_{12}^{1}=t_{12}^{2}=-t$, where $t \geq 1$. In the second equilibrium, the link is not formed because both players expect the other to make an unreasonable demand.

Note that the equilibrium supporting the empty network survives an elimination of weakly dominated strategies and is also a trembling hand perfect equilibrium. ${ }^{10}$ To eliminate this equilibrium using standard refinements would require the machinery of iterative elimination of strategies, which is cumbersome in games with a continuum of actions.

\footnotetext{
${ }^{10}$ Demanding $-t$ fares well in the case where the other agent happens to offer at least $t$.
} 
Alternatively, we should expect players forming a link to be able to coordinate their actions on that formation, as the real-life process that we are modeling would generally already involve some form of direct communication. This suggests a very simple refinement.

Given $t$, let $t_{-i j}$ indicate the vector of transfers found simply by deleting $t_{i j}^{i}$ and $t_{i j}^{j}$.

A vector $t$ is a pairwise equilibrium of one of the above games if it is an equilibrium of the game, and there does not exist any $i j \notin g(t)$, and $\widehat{t}$ such that

(1) $\pi_{i}\left(t_{-i j}, \widehat{t}_{i j}^{i}, \widehat{t}_{i j}^{j}\right) \geq \pi_{i}(t)$

(2) $\pi_{j}\left(t_{-i j}, \widehat{t}_{i j}^{i}, \widehat{t}_{i j}^{j}\right) \geq \pi_{j}(t)$, and

(3) at least one of (1) or (2) holds strictly. ${ }^{11}$

This refinement allows any two agents who have not yet formed a link to change their demands and offers in order to add a link. We focus attention on the addition of links, as players can already unilaterally choose to sever links by increasing their demands. Hence, the proper incentives to sever links are already captured by Nash equilibrium. ${ }^{12}$

While it is clear that Nash equilibria always exist in all the games we consider (the empty network is always supported in equilibrium), the existence of pairwise equilibria is not guaranteed. The following example shows that there exist environments for which no pairwise equilibrium exists.

Example 2 Nonexistence of Pairwise Equilibria

\footnotetext{
${ }^{11}$ Given the continuity of transfers, this is easily seen to be equivalent to requiring that both (1) and (2) hold strictly.

${ }^{12}$ There are many other refinements we could also consider. In the indirect transfer game, it seems natural to allow all agents to change their transfers on a given link, and we do introduce this more stringent refinement later. However, we believe that these more stringent refinements are harder to justify. Once one allows for such group deviations, it makes sense to go all the way to allowing general group deviations. At that point one is led to something that is equivalent to the concept of strong stability with side payments of Jackson and van den Nouweland (2000). Such a refinement is quite stringent, and while it has the nice property of only supporting efficient networks, it only applies in situations where there is substantial room for communication between all individuals.
} 

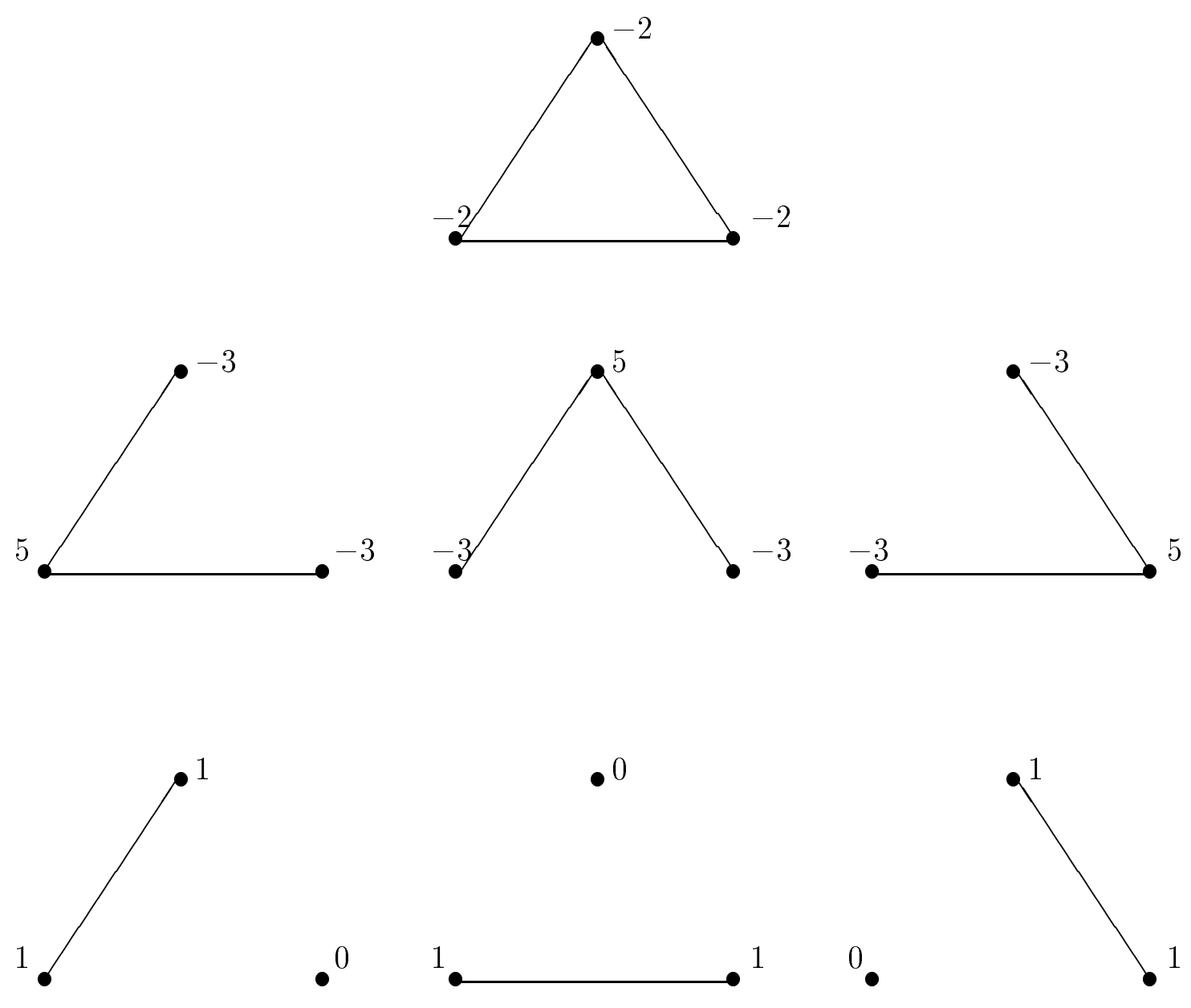

The empty network is a (pure strategy Nash) equilibrium, but not a pairwise equilibrium: two players can set zero demands to form a link and get $1>0$. No network that has at least two links can be supported as an equilibrium. Any such network must involve a player who gets a negative payoff, and who could profitably deviate by setting high demands on all his links which results in a payoff of 0 . Finally, a network with one link cannot be a pairwise equilibrium. The unlinked player and either of the linked players would benefit from setting transfers -3.5 and 3.5 , respectively.

\section{The Direct Transfer Game}

We now provide an analysis of the direct transfer network formation game. This is a natural, and the simplest, game to capture direct bargaining in the formation of links. 
We start with a simple example to show that externalities may prevent the emergence of an efficient network in equilibrium.

Example 3 Inefficient Network Formation with Direct Transfers and Positive Externalities

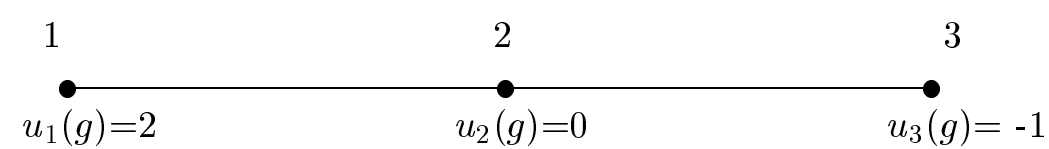

All other networks result in a utilities of 0 for all players.

The efficient network is the line $\{12,23\}$. For this network to be supported, we must have $t_{23}^{3} \leq-1$, as otherwise 3 would benefit by lowering $t^{3}$. If $t_{23}^{2} \geq 1 \geq-t_{23}^{3}$, player 2 will benefit by lowering $t_{23}^{2}$, regardless of what other links have formed as $u_{2}$ is 0 for all other networks. Thus, the network $\{12,23\}$ cannot be supported in equilibrium.

This example shows that, in the presence of positive externalities, direct transfers may be insufficient to guarantee that efficient networks are supported in equilibrium. In fact, this example clearly suggests that indirect transfers (in the form of link subsidization) are needed to support efficient networks in equilibrium.

The next example shows that, even in the absence of any externalities, the efficient network may fail to form in equilibrium.

Example 4 The Efficient Network is Not Supportable in the Complete Absence of Externalities.

Consider a three-player society and a profile of utility functions described as follows. Any player gets a payoff of 0 if he or she does not have any links. Player 1 gets a payoff of 2 if she has exactly one link, and a payoff of 1 if she has two links. Player 2 gets a payoff of -2 if he has exactly one link, and a payoff of 0 if he has two links. Player 3's payoff function is similar to that of player 2: he gets a payoff of -2 if he has exactly one link, and a payoff of 0 if he has two links.

It is clear from this specification that all players' payoffs depend only on the configuration of their own links and so there are no externalities in payoffs. This payoff structure is pictured in the network below. 

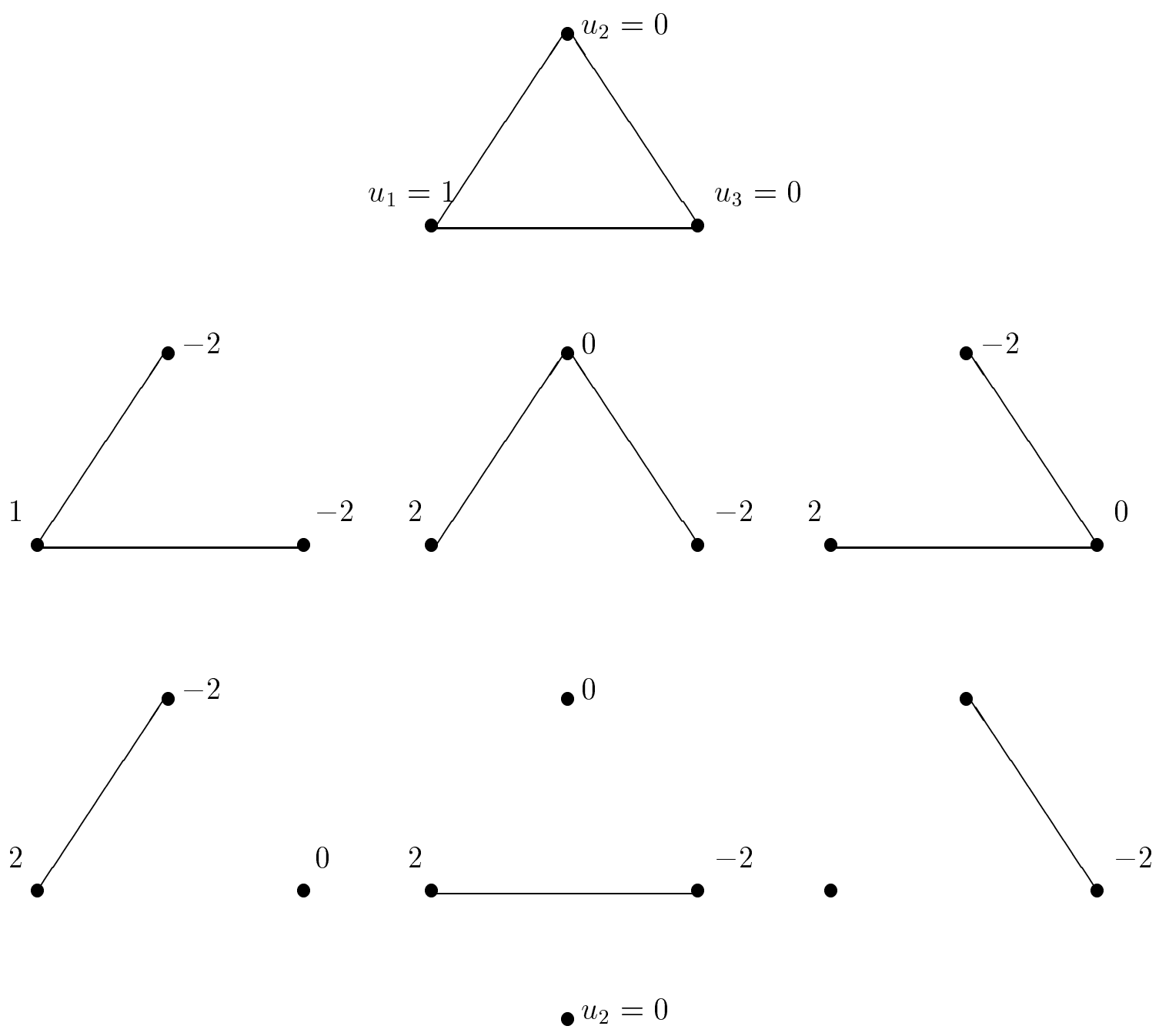

$u_{1}=0$

$u_{3}=0$

Let us argue that there is no equilibrium of the direct transfer game that supports the complete network, which is the unique efficient network. By setting $t_{2 i}^{2} \leq 0$ for each $i$, player 2 gets a payoff of at least 0 . The same is true for player 3 . Thus, players 2 and 3 must have a payoff of at least 0 in any equilibrium. Now, suppose by contradiction that the complete network were supported in an equilibrium. It would follow that $t_{1 i}^{1} \geq 0$ for at least one $i$, or otherwise one of players 2 and 3 would have a negative payoff. Without loss 
of generality, suppose that $t_{12}^{1} \geq 0$. Player 1 's payoff would then be $1-t_{12}^{1}-t_{13}^{1}$. Suppose that player 1 deviated and changed $t_{12}^{1}$ so that $t_{12}^{1}+t_{12}^{2}<0$. Then the network that would form would be 13,23 and player 1's payoff would become $2-t_{13}^{1}$ which is greater than $1-t_{12}^{1}-t_{13}^{1}$ (since $t_{12}^{1} \geq 0$ ). Hence player 1 would have a profitable deviation, and the complete network cannot be supported in equilibrium.

This example points to another difficulty in sustaining efficient networks. Players can choose to delete any combination of links. In order to sustain a given network as an equilibrium, it must be that each possible deviation is unprofitable, and each combination of links that could be deleted might require different transfers in order to be avoided. Some of these combinations might be in conflict with each other. In the above example, it is the possibilities that either player 2 or 3 might sever both of his links that lies in conflict with what player 1 can get by severing a single link at a time.

The preceding examples suggest two features that the link formation game must have in order to always result in efficient networks in equilibrium. First, indirect transfers are needed in order to take care of externalities, as suggested by Example 3. Second, as Example 4 suggests, transfers need to be contingent on the network in order to adjust to the particular combination of links that are formed.

Before turning to a full analysis of the games with indirect transfers and/or contingent transfers, we analyze the game with only direct transfers. We do this for several reasons. First, there may be applications where this is the most appropriate game; second, this serves as a useful benchmark; and third, if an efficient network can be supported via just direct transfers, then it is in a sense more plausible that it will emerge than one that requires a more involved transfer scheme to sustain it.

We first offer a complete characterization of the networks that can be supported in equilibrium of the direct transfer game, and then we identify some settings where direct transfers suffice to support efficient networks.

\section{A Complete Characterization of Networks Supported by Direct Transfers: The Network Balance Condition}

A set of nonnegative weights $\left\{\mu_{\ell}^{i}\right\}_{i \in N, \ell \subset L_{i}(g)}$ is balanced relative to a network $g$ if

$$
\sum_{\ell \subset L_{i}(g): i j \in \ell} \mu_{\ell}^{i}=\sum_{\ell \subset L_{j}(g): i j \in \ell} \mu_{\ell}^{j}
$$

for each $i j \in g$.

The network $g$ is balanced relative to the profile of utility functions $u$ if

$$
\sum_{i} \sum_{\ell \subset L_{i}(g)} \mu_{\ell}^{i} m u_{i}(g, \ell) \geq 0
$$


for every balanced vectors of weights.

We should emphasize that the balance conditions identified here are different both in structure and implications from the balance conditions used in cooperative game theory. Our balance condition assigns weights to each player and combination of bilateral links involving that player. This contrasts with weights assigned to coalitions in cooperative games, and reflects the bilateral structure of networks. This also reflects the fact that these balance conditions are set to address an equilibrium notion that deals with deviations by at most two individuals at a time.

Proposition 5 A network $g$ is supportable as an equilibrium of the direct transfer network formation game relative to the profile of utility functions $u$ if and only if it is balanced relative to the profile of utility functions $u$.

The proof of Proposition 5, together with all of our other proofs, appears in the appendix. It follows a logic similar to that of the proof of the existence of the core for balanced games, exploiting duality to convert the problem of existence of transfers into a set of balance conditions. There are a couple of twists due to the bilateral nature of the problem, but the proof is fairly short. While balance conditions are not transparent to interpret, they still have a simple intuition. They examine whether or not all of the possible marginal utilities from potential deviations can be overcome via some set of transfers. Our balance conditions prove useful in exploring sufficient conditions for efficient networks to be supported in equilibrium.

Proposition 5 only characterizes supportability, and not supportability via pairwise equilibrium. Clearly this provides necessary, but not sufficient conditions for supportability via pairwise equilibrium. The additional constraints imposed by pairwise equilibrium are difficult to capture through balancedness conditions. Nevertheless, we can identify a sufficient condition, as follows.

Proposition 6 If a network $g$ is supportable via pairwise equilibrium by the direct transfer network formation, then it is balanced relative to the profile of utility functions $u$. Conversely, if $u$ satisfies nonnegative externalities, and $g$ is efficient and balanced relative to $u$, then $g$ is supportable via pairwise equilibrium by the direct transfer network formation game.

More generally, we show the following lemma, which also applies to the indirect transfer game.

Lemma 7 If $g$ is efficient and supportable via the direct or indirect transfer game, and $u$ satisfies nonnegative externalities, then $g$ is supportable in pairwise equilibrium.

\section{Supportability with Nonpositive Externalities and Convexity in Own-Links}


We now identify sufficient conditions for the efficient network to be supported in equilibrium, using the intuition of Examples 3 and 4. Example 3 suggests that we should look at situations where externalities are nonpositive. Example 4 suggests a restriction that marginal payoffs from a given set of links be at least as high as the sum of the marginal payoffs from separate links. This condition is formalized as follows.

A profile of utility functions $u$ are convex in own-links if

$$
m u_{i}(g, \ell) \geq \sum_{i j \in \ell} m u_{i}(g, i j)
$$

for all $i, g$, and $\ell \subset L_{i}(g)$.

Under these two conditions efficient networks are supportable, as stated in the following proposition.

Proposition 8 If utility functions are convex in own-links and satisfy nonpositive externalities, then any efficient network $g$ is supportable via the direct transfer game. If utility functions are convex in own links and satisfy no externalities, then $g$ is supportable via a pairwise equilibrium. ${ }^{13}$

Goyal and Joshi (2003)'s model of networks of collaboration in oligopoly provides an example of a setting where convexity in own links and nonpositive externalities hold. Suppose that $n$ firms are engaged in quantity competition in a market for a homogeneous good. By forming a link, firms can decrease their constant marginal cost of production. Suppose that cost reductions are an increasing but concave function of the number of links, $c\left(\eta_{i}(g)\right)$ where $\eta_{i}(g)$ denotes the number of edges of firm $i$ in the graph $g$. It is easy to check that the formation of links by players $j$ and $k$ reduces the production costs of those two firms, resulting in a decrease in the profit of firm $i$ and so there are nonpositive (negative) externalities. Furthermore, when the additional benefit of a new link is decreasing with the number of links the firm has already formed, convexity in own links holds. Thus, Proposition 8 applies and the efficient network is supportable via the direct transfer game.

\section{Link-Separable Payoffs}

\footnotetext{
${ }^{13}$ Toni Calvo-Armengol has pointed out to us that this proposition holds if we weaken convexity in own-links to only require that there exist some $\alpha>0$ such that $m u_{i}(g, \ell) \geq \alpha \sum_{i j \in \ell} m u_{i}(g, i j)$ for all $i, g$, and $\ell \subset L_{i}(g)$. [The proof in the appendix is easily modified, by simply placing an $\alpha$ on the right hand side of the inequalities.] This captures some applications, such as the co-author model of Jackson and Wolinsky (1996), which satisfies nonpositive externalities and the $\alpha$ version of convexity in own links, but does not satisfy convexity in own links. We have not stated the proposition using this weaker convexity condition, as Proposition 13, which uses a parallel convexity condition cannot be stated in the weaker form.
} 
While Proposition 8 shows that the efficient network is supported as one equilibrium of the game, it does not guarantee that no other networks will be supported as equilibria as well. In order to check when efficient networks may be supported as the only pairwise equilibria of the direct transfer game, we turn to a special case of convexity in own links, where payoffs are separable across links.

Payoffs are link-separable, if for each player $i$ there exists a vector $w^{i} \in \mathbb{R}^{n-1}$, where $w_{j k}^{i}$ is interpreted as the net utility that player $i$ obtains from link $j k$ forming. Then

$$
u_{i}(g)=\sum_{j k \in g} w_{j k}^{i}
$$

This very strong condition states that players view relationships completely separately. A special case of link separable payoffs is one where agents only care about their direct links.

Corollary 9 If payoffs are link-separable and have nonpositive externalities, then any efficient network $g$ is supportable via the direct transfer game. Furthermore, if payoffs are link-separable and have no externalities, then $g$ is supportable via a pairwise equilibrium if and only if $g$ is efficient.

The first statement and first part of the second statement follow from Proposition 8. To see the only if claim, suppose to the contrary that $g$ is supportable via a pairwise equilibrium but not efficient. Then there exists $g^{\prime}$ such that $\sum_{i} u_{i}\left(g^{\prime}\right)>\sum_{i} u_{i}(g)$. As payoffs are link separable and have no externalities, either there exists $i j \in g \backslash g^{\prime}$ such that $w^{i} i j+w^{j} i j<0$ or there exists $i j \in g^{\prime} \backslash g$ and $w^{i} i j+w^{j} i j>0$. In the first case, $g$ cannot be supported as an equilibrium, because one of the two players has an incentive to increase her demanded transfer thereby severing the link; in the second case, $g$ cannot be supported as a pairwise equilibrium, since will exist a pair of compatible transfer such that the players have an incentive form the link.

\section{Distance-Based Payoffs and Stars}

Convexity in own links and nonpositive externalities are sufficient conditions for the efficient network to be supported as an equilibrium of the direct transfer game, but are by no means necessary, as there are other conditions that ensure that network balance is satisfied. We now exhibit another class of utility functions, which violate both these conditions, but for which the efficient network can be sustained in equilibrium. This is the class of distance based utilities, where players get value from the number of players they are linked to, and this value is decreasing with the distance of the connection.

Let $d(i, j)$ denote the distance between $i$ and $j$ in terms of the number of links in the shortest path between them (setting $d(i, j)=\infty$ if there is no path). 
A profile of utility functions is distance-based if there exist $c$ and $f$ such that

$$
u_{i}(g)=\sum_{j \neq i} f(d(i, j))-c\left|L_{i}(g)\right|
$$

for all $i$, where $c \geq 0$ is a cost per link, and $f$ is a nonincreasing function.

A distance-based payoff structure is one where players may get benefits from indirect connections, but where those benefits are determined by the shortest paths. Special cases of distance-based payoffs are the connections model and truncated connections models of Jackson and Wolinsky (1996). In such settings, "star" networks play a very central role, as captured in the following proposition.

Proposition 10 If $u$ is distance-based, then the unique efficient network structure is

(i) the complete network $g^{N}$ if $c<f(1)-f(2)$,

(ii) a star encompassing all players if $f(1)-f(2)<c<f(1)+\frac{(n-2) f(2)}{2}$, and

(iii) the empty network if $f(1)+\frac{(n-2) f(2)}{2}$.

In the case where $c$ is equal to $f(1)-f(2)$ or $f(1)+\frac{(n-2) f(2)}{2}$, there are can be a variety of network structures that are efficient. Nevertheless, the star is still efficient in those cases.

The proof of Proposition 10 is an easy extension of the proof of a Proposition in Jackson and Wolinsky (1996), but we include it in the appendix for completeness.

It turns out that efficient networks can be supported (even by pairwise equilibrium) in the direct transfer game for distance-based payoff structures. This result is related to the special nature of the efficient network. In a star, every player is related to the center and positive externalities pass through the center. Peripheral players can subsidize the center of the star to keep their links formed, and this properly accounts for externalities. This is captured in the following corollary to Propositions 5 and 6 .

Corollary 11 If $u$ is distance-based, then some efficient network is supportable as an equilibrium the direct transfer game, and is also supportable in pairwise equilibrium.

The claim is easy to see directly in cases where either the empty or complete networks are efficient. Consider the remaining case where $f(1)-f(2) \leq c \leq f(1)+\frac{(n-2) f(2)}{2}$, and thus a star involving all players is efficient. Here, we let us discuss how one can verify the balance conditions. An agent $i$ connected to the center $j$ in a star has only one link, we can simply set $\mu_{\{i j\}}^{i}=c$ for any $c \geq 0$. Then for the center $j$, it must be that $\sum_{\ell \subset L_{j}(g): i j \in \ell} \mu_{\ell}^{j}=c$. The fact that a star is balanced then follows from noting that $c m u_{i}(g, i j)+c \mu_{j}(g, i j)=2 f(1)+(n-2) f(2)-2 c \geq 0$ in situations where the 
star is efficient, and noting that the center's payoff is additively separable across links. ${ }^{14}$ Proposition 6 implies that we can support an efficient $g$ as a pairwise equilibrium, noting that there are nonnegative externalities in a distance-based $u$ (as adding a link that does not involve $i$ can only increase $i$ 's payoff as it may decrease the distance between $i$ and some other agent, but does not impose a cost on $i$ )

\section{Indirect Transfers}

As discussed above, indirect transfers are needed to overcome some of the difficulties linked to positive externalities in the network. However, in the indirect transfer game, convexity in own-links is no longer sufficient to overcome the difficulty due to the deletion of combinations of links, as a player's deviation can result in the severance of links in which he is not involved. Thus the problem associated with the interaction of the multitude of bilateral relationships is more complex when indirect transfers are present. This is illustrated in the following example.

Example 12 Efficient Network are not Supportable with Indirect Transfers and Convexity in Own-Links

Consider a three-player society with payoffs pictured below.
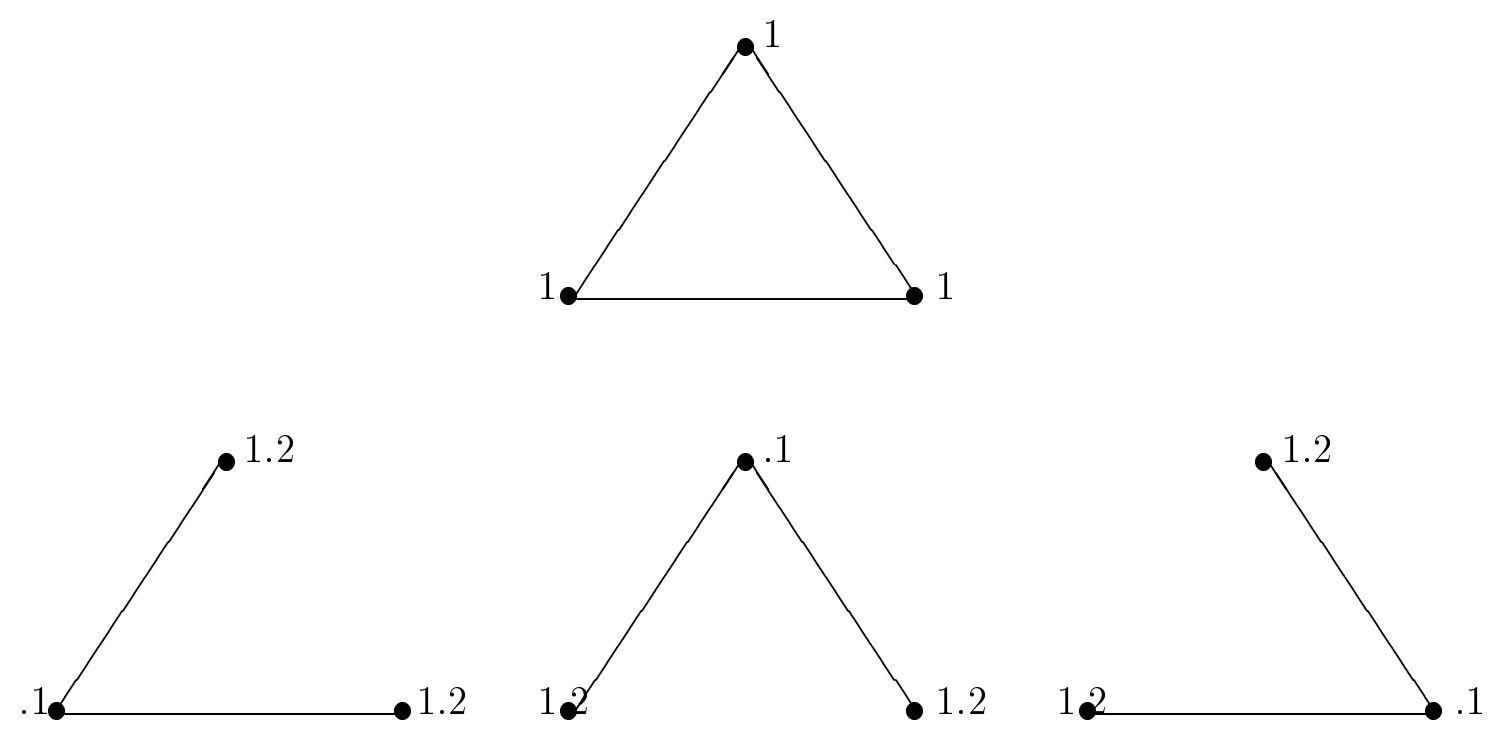

\footnotetext{
${ }^{14}$ This also gives us an idea of which transfers support a star as an equilibrium with agent 1 as the center. Setting $t_{1 i}^{i}=f(1)+(n-2) f(2)-c, t_{j i}^{i}=-(n-1) f(1)$ for $j>1$, and $t_{1 i}^{1}=-[f(1)+(n-2) f(2)-c]$ for each $i$. It is easily seen that these form an equilibrium that supports the star.
} 

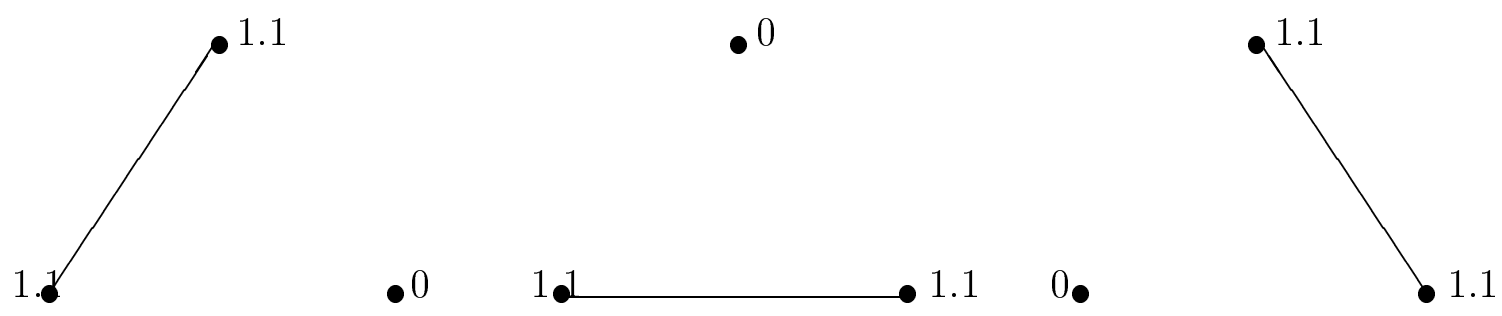

The complete network is efficient but is not the outcome of any equilibrium of the indirect transfer network formation game. Consider any player $i$. Player $i$ must offer to subsidize the link $j k$ by an amount of at least .4, as otherwise at least one of $j$ and $k$ will have an incentive to "sever" the link (set their demand to be less than -.2 ).

Consider some player $i$ and link $i j$ such that $t_{i j}^{i} \geq 0$. Such a link must exist if the complete network is supported. Consider the following deviation: player $i$ reduces the payment on the link $j k$ and "severs" link $i j$ (setting $t_{i j}^{i}$ to be low enough so that $i j$ does not form). In that case, the only link formed is link $i k$, and player $i$ 's base payoff is the increased, and transfers have decreased which is strict improvement for player $i$.

The above network is convex in own-links, as the marginal utility of any second ownlink is negative while the marginal utility of any set of two own-links is always positive. However, note that the convexity in links fails more generally. The marginal utility to player 1 at the complete network of the links 12,23 is negative, while the marginal utility of 23 at the complete network is 1.1, and the marginal utility of 12 is -.2 , so the sum of the marginal utilities is positive. Indeed, this is the source of the problem in the example.

\section{Convexity in All Links}

A profile of utility functions $u$ is convex in all links if

$$
m u_{i}(g, \ell) \geq \sum_{j k \in \ell} m u_{i}(g, j k)
$$

for all $i, g$, and any $\ell \subset g$.

We can now state the following proposition.

Proposition 13 If payoffs are convex in all links, then any efficient network $g$ is supportable via the indirect transfer game. If payoffs also have nonnegative externalities, then $g$ is supportable via pairwise equilibrium. 
With indirect transfers, efficient networks can thus be supported irrespective of the nature of externalities in payoffs if one simply requires equilibrium, and can be supported under nonnegative externalities if we require pairwise equilibrium. However, the convexity assumption property needed to support efficient networks is stronger than "convexity in own links" which was required to support efficient networks in the direct transfer game. In words, we require that the marginal benefit of any subset of links (and not only the links involving the player) be greater than the sum of the additional benefits link per link. This convexity assumption is likely to be satisfied when the marginal benefit of a new link is decreasing with the number of links already formed. Examples of such situations are trading and information sharing networks. In these networks, the addition of new connections typically has positive externalities on all the players. All players benefit from enlarging the set of trading opportunities, or increasing the number of communication channels. However, the marginal benefit of an additional link will often be decreasing with the number of links already formed. If players incur a cost for forming direct links, the efficient network (typically the complete network) may not be formed at equilibrium, because players do not internalize the positive externalities they produce on other players. We claim that indirect transfers will allow for the formation of the complete network in such trading and information sharing networks.

While indirect transfers enable the support of efficient networks as equilibria of the game, there is no guarantee that efficient networks are the only equilibria of the game. We now show that, in games with link separable payoffs and nonnegative externalities, efficient networks are the only equilibria of the game if we allow cooperation by all players in the formation of additional links. More precisely, we strengthen the definition of pairwise equilibrium to allow all players to change their offers/demands on a given link.

A vector $t$ is a strong pairwise equilibrium of the indirect transfer game if it is an equilibrium of the game, and there does not exist any ij $\notin g(t)$ and $S \subset N$, and $\hat{t}$ that differs from $t$ only on $t_{i j}^{k}$ where $k \in S$, and such that $\pi_{i}\left(t_{-i j}, \widehat{t}_{i j}\right) \geq \pi_{i}(t)$, for all players $i \in S$, with strict inequality for some of the players.

This definition is weaker than a strong equilibrium, where arbitrary subsets of players can alter all of their strategies. We work with the weaker definition since the Corollary below still holds for this weaker definition. In fact, it turns out that under link separability and nonnegative externalities, the strong equilibria and the strong pairwise equilibria of the indirect transfer game coincide. This is easy to see as the payoffs separate completely across links, and so one can consider links one at a time.

Corollary 14 If payoffs are link-separable and satisfy nonnegative externalities, then $g$ is supportable via a strong pairwise equilibrium of the indirect transfer game if and only if $g$ is efficient. 


\section{Network Contingent Transfers}

We now consider network formation games where players can condition their transfers on the network that is formed.

As we see now, allowing transfers to be contingent on the network that forms has a big impact on the set of networks that can be supported as equilibrium networks, even when only direct transfers are possible. To understand why contingent transfers may help to support efficient networks, even when only direct transfers are possible, reconsider Example 3. In that example, the efficient network could not be formed in the direct transfer game, and we argued that the efficient network could be supported if indirect transfers were allowed, as player 1 needs to subsidize the formation of link 23. There is another possibility, which does not require the use of indirect transfers, but instead relies on contingent transfers. Player 1 could make transfers to player 2, to pass them on to player 3. The difficulty is that if player 1 makes this transfer to player 2, then player 2 might not form the link with player 3 and keep the transfer. This can be rectified if transfers can be made contingent on the network that forms.

More generally, contingent direct transfers can be built up along paths so that they end up moving as if they were indirect transfers within connected components. This insight is the key to the following proposition and corollary.

Proposition 15 Consider the contingent version of the direct transfer game and any $u$. There exists an equilibrium where the network $g$ is formed and the payoffs are $y \in \mathbb{R}^{n}$ where $y_{i} \geq 0$ for all $i \in N(g)$ if and only if $\sum_{i \in N\left(g^{\prime}\right)} u_{i}(g)=\sum_{i \in N\left(g^{\prime}\right)} y_{i}$ for all $g^{\prime} \in C(g)$, and $y_{i} \neq u_{i}(g)$ implies $i \in N(g)$.

Corollary 16 Consider the contingent version of the direct transfer game. Consider any $u$ and network $g$ such that $\sum_{i \in N\left(g^{\prime}\right)} u_{i}(g) \geq 0$ for all components $g^{\prime} \in C(g)$. There exists an equilibrium supporting $g$. Moreover, there is an equilibrium corresponding to each allocation $y \in \mathbb{R}^{n}$ such that $\sum_{i \in N\left(g^{\prime}\right)} u_{i}(g)=\sum_{i \in N\left(g^{\prime}\right)} y_{i}$ for each $g^{\prime} \in C(g)$ and $y_{i}=u_{i}(g)$ or $y_{i}<0$ implies $i \notin N(g)$.

Proposition 15 is based on a constructive proof, where we explicitly derive equilibrium contingent transfers to support the network. While this proposition shows that a wide set of networks can be supported as equilibria of the contingent direct transfer game, it is limited by the fact that transfers cannot flow across separate components of a network in the direct transfer game, even if payments are contingent. If we allow for contingent indirect transfers, then there are additional networks that can be supported, as we now show.

Proposition 17 Consider the contingent version of the indirect transfer network formation game. Consider any $u$, any network $g$, and any allocation $y \in \mathbb{R}_{+}^{n}$ such that 
$\sum_{i} y_{i}=\sum_{i} u_{i}(g)$, and $y_{i}>u_{i}(g)$ implies $i \in N(g) .{ }^{15}$ There exists an equilibrium where $g$ is formed and payoffs are $y$.

Corollary 18 Consider the contingent version of the indirect transfer network formation game, and any $u$. Any efficient network such that disconnected players earn zero payoffs is supportable. Moreover, there is an equilibrium supporting each allocation $y \in \mathbb{R}_{+}^{n}$ such that $\sum_{i} y_{i}=\sum_{i} u_{i}(g)$ and $y_{i}>0$ implies $i \in N(g)$.

Proposition 17 and Corollary 17 show that the combination of indirect transfers and allowing these to be contingent allows the support of almost all efficient networks as equilibria. The artifact that this includes situations where negative externalities might be present is due to the fact that we are considering only equilibrium and not pairwise equilibrium.

\section{Pairwise Equilibria with Contingent Transfers}

Propositions 15 and 17 have counterparts for pairwise equilibrium, ${ }^{16}$ provided the network being supported is efficient and there are nonnegative externalities. A simple extension of the proof Lemma 7 leads to the following corollary.

Corollary 19 Consider the contingent version of the indirect transfer network formation game, and any u satisfying nonnegative externalities. Consider the contingent version of the indirect transfer network formation game. Consider any efficient network $g$ and allocation $y \in \mathbb{R}_{+}^{n}$ such that $\sum_{i} y_{i}=\sum_{i} u_{i}(g)$, and $y_{i}>u_{i}(g)$ implies $i \in N(g)$. Then $g$ is supportable as a pairwise equilibrium with equilibrium payoffs $y$.

\section{Transfers to Prevent Link Formation}

The previous analysis shows that efficient networks can be supported as a Nash equilibrium of the indirect contingent transfer game under very mild assumptions on the payoff function. However, in order to sustain efficient networks as pairwise equilibria, we needed the additional restriction that externalities are nonnegative. To see why this is important, consider the following example exhibiting negative externalities.

Example 20 Negative Externalities and Inefficient Pairwise Equilibria

\footnotetext{
${ }^{15}$ The $y$ 's in Proposition 17 are required to be nonnegative. One can also support the networks from Proposition 15 that are not covered in this proposition through the construction used there. The difference is that here one sometimes needs a player not in $N(g)$ to subsidize the formation of a component that has a negative value to its members. For this to work, it must be that the disconnected player earns a nonnegative payoff, or they would withdraw their subsidies. Rather than break this into separate cases, we have simply worked with the assumption of nonnegative payoffs.

${ }^{16}$ In order to define pairwise equilibrium, allow players $i$ and $j$ to vary their announcements $t_{i j}^{i}(\cdot)($ as contingent on any network).
} 
The society has four players. If one link forms, the two players involved each get a payoff of 3 .
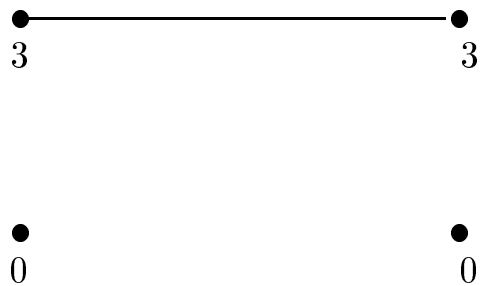

If two (separate) links form, then the four players each get a payoff of 1 .
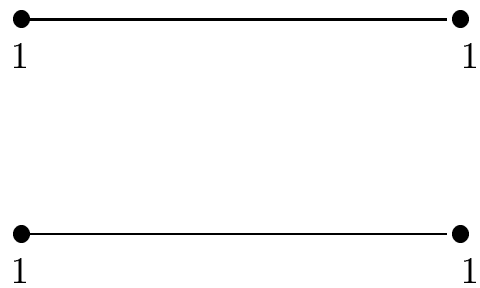

All other networks result in a payoff of 0 .

In this example, the only pairwise equilibria are inefficient. ${ }^{17}$ Two players who are disconnected always benefit from forming a link, and there is no way to prevent them from doing so. Indeed, two players involved in a link would like to pay the other players not to form a link.

\section{A Game with Payments to Prevent Link Formation}

In order to overcome the difficulty exhibited in Example 20, we need to have a game where players have the ability to make transfers to prevent the formation of links.

We first describe a game that allows payments to prevent link formation, but without considering contingent transfers. We come back to incorporate contingencies after this game is made clear. The game is based on the indirect link formation game, with the following modification. Each player announces two transfers per link, instead of just

\footnotetext{
${ }^{17}$ The efficient network is supportable as an equilibrium, where the two disconnected players fail to form a link because each demands too large a transfer. This, again, is a case where pairwise equilibrium is a reasonable refinement.
} 
one. This pair of announcements by player $i$ relative to link $j k$ is denoted $t_{j k}^{i+}$ and $t_{j k}^{i-}$. Again, these must be nonnegative if $i \notin j k$, and can be anything otherwise. Player $i$ also announces $m_{j}^{i} \in\{+,-\}$ for each $j \neq i$. The interpretation is that $i$ is declaring whether the default decision on link $i j$ is not to add $i j$ or to add $i j$.

The network $g(t, m)$ is then determined as follows.

- If $m_{j}^{i} \neq m_{i}^{j}$, then $i j \notin g$.

- If $m_{j}^{i}=m_{i}^{j}=+$, then $i j \in g$ if and only if $\sum_{k} t_{i j}^{k+} \geq 0$.

- If $m_{j}^{i}=m_{i}^{j}=-$, then $i j \notin g$ if and only if $\sum_{k} t_{i j}^{k-} \geq 0$.

Payoffs are then

$$
u_{i}(g(t))-\sum_{j k \in g(t), m_{k}^{j}=m_{j}^{k}=+} t_{j k}^{i+}-\sum_{j k \notin g(t), m_{k}^{j}=m_{j}^{k}=-} t_{j k}^{i-} .
$$

The contingent version of the game with payments to prevent the formation of links is the version where the $t^{i}$ and $m_{j}^{i}$, s are announced as a function of $g$, and then solved via an ordering over games, just as before.

Equilibrium is again pure strategy Nash equilibrium in pure strategies, and pairwise equilibrium and strong pairwise equilibrium are the obvious extensions to this game. In particular, here a pairwise equilibrium is an equilibrium such that no pair $i$ and $j$ could alter their strategies pertaining to $i j$ (as contingent on any $g$ 's $m_{j}^{i}(\cdot), m_{i}^{j}(\cdot), t_{i j}^{i+}(\cdot)$, $\left.t_{i j}^{j+}(\cdot), t_{i j}^{i-}(\cdot)\right)$ and both be weakly better off and one strictly better off. A strong pairwise equilibrium is an equilibrium such that there does not exist any $i j$ and a deviation by some set of players $S \subset N$ on the strategies $t_{i j}^{k+}(\cdot), t_{i j}^{k-}(\cdot)$, (and $m_{j}^{i}(\cdot)$ if $k \in i j$ ) such that all members of $S$ are strictly better off as a result of the deviation.

To see how the game defined above works, reconsider Example 20.

Example 21 Negative Externalities with Payments to Prevent Links

Consider the payoff function of Example 20. Let us find a pairwise equilibrium of the game with payments not to form links that supports an efficient network. Let us support the efficient network $\{12\}$. Have all players set $t_{12}^{i+}(\{12\})=0$. Set $t_{34}^{1-}(\{12\})=$ $t_{34}^{2-}(\{12\})=1 / 2$ and $t_{34}^{3-}(\{12\})=t_{34}^{4-}(\{12\})=-1 / 2$, and $m_{34}^{3}(g)=m_{34}^{4}(g)=-$ for all $g$, and $m_{i j}^{i}(g)=+$ otherwise. For any other transfers set $t_{i j}^{i \cdot}(g)=-2$, and $t_{j k}^{i \cdot}(g)=0$ when $i \notin j k$.

Here, players 1 and 2 pay players 3 and 4 if the link 34 is not formed. It is straightforward to check that this is a pairwise equilibrium. 
Proposition 22 In the contingent game with indirect transfers to form or not to form links, any efficient network is supportable via pairwise equilibrium, and in fact via strong pairwise equilibrium.

Proposition 22 shows that with the ability to make contingent indirect transfers that both subsidize the formation or the prevention of links, efficient equilibria are supportable via pairwise equilibria.

\section{Concluding Remarks}

We have defined a series of games of network formation where transfers among players are possible, and through an analysis of the equilibrium networkse have shed light on how the type of transfers is related to the support of efficient networks. We pointed out two basic hurdles in supporting efficient networks in equilibrium. First, the presence of positive externalities in payoffs may prevent the formation of efficient networks, because players involved in a link do not internalize the external effects the link has on other players. Second, players may be unable to reach an efficient network because the transfers needed to prevent the deletion of various subsets of links may be incompatible. Overcoming positive externalities relies on players' ability to subsidize the formation of links by other players, and overcoming negative externalities relies on their ability to pay to prevent the formation of links. The problem of dealing with the combinatorial nature of the set of bilateral links that need to be considered together is overcome if players have the ability to condition their transfers on the entire network.

We would like to point out a limitation of our analysis. While some of our results provide complete characterizations of supportable networks (for instance, the network balance conditions, the link separability conditions, and the conditions outlined for the contingent direct transfer game); others only outline sufficient conditions for the support of efficient networks and rely on constructive proofs. This leaves open some questions of the precise necessary conditions for supportability in some of the games, which goes together with a question of which inefficient networks might emerge in some of the games. Closing the remaining gaps to developing a full understanding of the situations where efficient networks emerge as the unique plausible equilibria of a network formation game is a priority for future research.

\section{References}

Belleflamme, P. and Bloch, F. (2001) "Market Sharing Agreements and Stable Collusive Networks", forthcoming, International Economic Revie1

Calvo-Armengol, A. (2004) "Pairwise Stability and Nash Equilibria in Network Formation," mimeo: Universitat Autonoma de Barcelona. 
Currarini, S. and M. Morelli (2000) "Network Formation with Sequential Demands," Review of Economic Design, 5, 229-250.

Dutta, B. and S. Mutuswami (1997) "Stable Networks," Journal of Economic Theory, $76,322-344$.

Furusawa, T. and H. Konishi (2002) "Free Trade Networks," mimeo: Yokohama National University and Boston College.

Goyal, S. and S. Joshi (2000) "Bilateralism and Free Trade", mimeo., University of Essex and Georgetown University.

Goyal, S. and S. Joshi (2003) "Networks of Collaboration in Oligopoly", Games and Economic Behavior, 43, 57-85.

Jackson, M.O. (2003a). "The Stability and Efficiency of Economic and Social Networks," in Advances in Economic Design, edited by S. Koray and M. Sertel, Springer-Verlag: Heidelberg, and reprinted in Networks and Groups: Models of Strategic Formation, edited by B. Dutta and M.O. Jackson, Springer-Verlag: Heidelberg.

Jackson, M.O. (2003b). "A Survey of Models of Network Formation: Stability and Efficiency," forthcoming in Group Formation in Economics: Networks, Clubs, and Coalitions, edited by G. Demange and M. Wooders, Cambridge University Press: Cambridge. http://www.hss.caltech.edu/ jacksonm/netsurv.pdf

Jackson, M.O. and van den Nouweland, A. (2000) "Strongly Stable Networks," forthcoming: Games and Economic Behavior, http://www.hss.caltech.edu/ jacksonm/coopnet.pdf Jackson, M.O. and A. Wolinsky (1996) "A Strategic Model of Social and Economic Networks," Journal of Economic Theory, 71, 44-74.

Matsubayashi, N. and S. Yamakawa (2004) "A Network Formation Game with an Endogenous Cost Allocation Rule," mimeo: NTT Communications.

Mutuswami, S. and E. Winter (2002) "Subscription Mechanisms for Network Formation," Journal of Economic Theory, .

Slikker, M. and van den Nouweland, A. (2001a) Social and Economic Networks in Cooperative Game Theory, Kluwer.

Slikker, M. and A. van den Nouweland (2001b) "A One-Stage Model of Link Formation and Payoff Division," Games and Economic Behavior, 34, 153-175.

Watts, A. (2001) "A Dynamic Model of Network Formation," Games and Economic Behavior, 34, 331-341. 


\section{Appendix A Pairwise Stability and Equilibrium Networks}

In this Appendix, we discuss the relation between the networks supported in the direct and indirect transfer games, and the pairwise stable networks introduced by Jackson and Wolinsky (1996). This discussion highlights the connections between situations where the allocation rule is fixed before the formation of the networks, and situations where players can freely bargain over the allocation of the value of additional links.

The following definitions identify networks that are stable when the payoffs are fixed before the formation process. ${ }^{18}$

A network $g$ is pairwise stable with respect to a profile of utility functions $u$ if

(i) for all $i$ and $i j \in g, u_{i}(g) \geq u_{i}(g-i j)$, and

(ii) for all $i j \notin g$, if $u_{i}(g+i j)>u_{i}(g)$ then $u_{j}(g+i j)<u_{j}(g)$.

This is a self-evident solution concept that requires that no player benefit by severing a link and no two players benefit by adding one.

A network $g$ is pairwise stable* with respect to a profile of utility functions $u$ if

(i) for all $i$ and $\ell \subset L_{i}(g), u_{i}(g) \geq u_{i}(g \backslash \ell)$, and

(ii) for all $i j \notin g$, if $u_{i}(g+i j)>u_{i}(g)$ then $u_{j}(g+i j)<u_{j}(g)$.

This variation on pairwise stability is stronger than pairwise stability in that it allows players to sever sets of links rather than just considering one link at a time. This solution is discussed by Jackson and Wolinsky (1996) and is also essentially the same as the pairwise Nash equilibrium refinement of pairwise stability discussed by Goyal and Joshi $(2003) .^{19}$

The next definition is a way of incorporating transfers into the study of network formation without actually modeling the bargaining process explicitly. ${ }^{20}$

A network $g$ is pairwise stable with transfers with respect to a profile of functions $u$ if

\footnotetext{
${ }^{18}$ The first two definitions are from Jackson and Wolinsky (1996). Strong pairwise stability is discussed by Jackson and Wolinsky (1996, section 5), but is not named.

${ }^{19}$ For a more in depth discussion of the relation between the concepts of pairwise stable and pairwise stable*, see Calvo-Armengol (2004). We stay away from the term pairwise Nash equilibrium, to avoid confusion with pairwise equilibrium.

${ }^{20}$ This differs from the concept of pairwise stability allowing for side payments that is discussed by Jackson and Wolinsky (1996). That concept had a stronger requirement in (i), requiring that $u_{i}(g) \geq$ $u_{i}(g-i j)$ and $u_{j}(g) \geq u_{j}(g-i j)$. If transfers are possible in sustaining a network, and not just in deviations, then arguably the definition here is more appropriate.
} 
(i) $i j \in g \Rightarrow u_{i}(g)+u_{j}(g) \geq u_{i}(g-i j)+u_{j}(g-i j)$, and

(ii) $i j \notin g \Rightarrow u_{i}(g)+u_{j}(g) \leq u_{i}(g-i j)+u_{j}(g-i j)$.

Part (ii) captures the idea that there are no two players who could add a link between them, together with some transfers, and both be better off. Part (i) captures the idea that if a link is in the network, then there must be some transfer (possibly 0) for which both players do not wish to delete the link.

While the notions of pairwise stability and pairwise stability* can differ from the equilibria of the direct transfer game, the notion of pairwise stability with transfers captures some of the spirit of the equilibria of the direct transfer game.

Proposition 23 The set of networks supportable as pairwise equilibria is exactly the intersection of those networks that are supportable via the direct transfer game and the networks that are pairwise stable with transfers.

The relationship between supportable networks, pairwise equilibria, and the other pairwise stability concepts is outlined in the following proposition. The relationships between the solution concepts 24 are captured in the following Venn diagram.

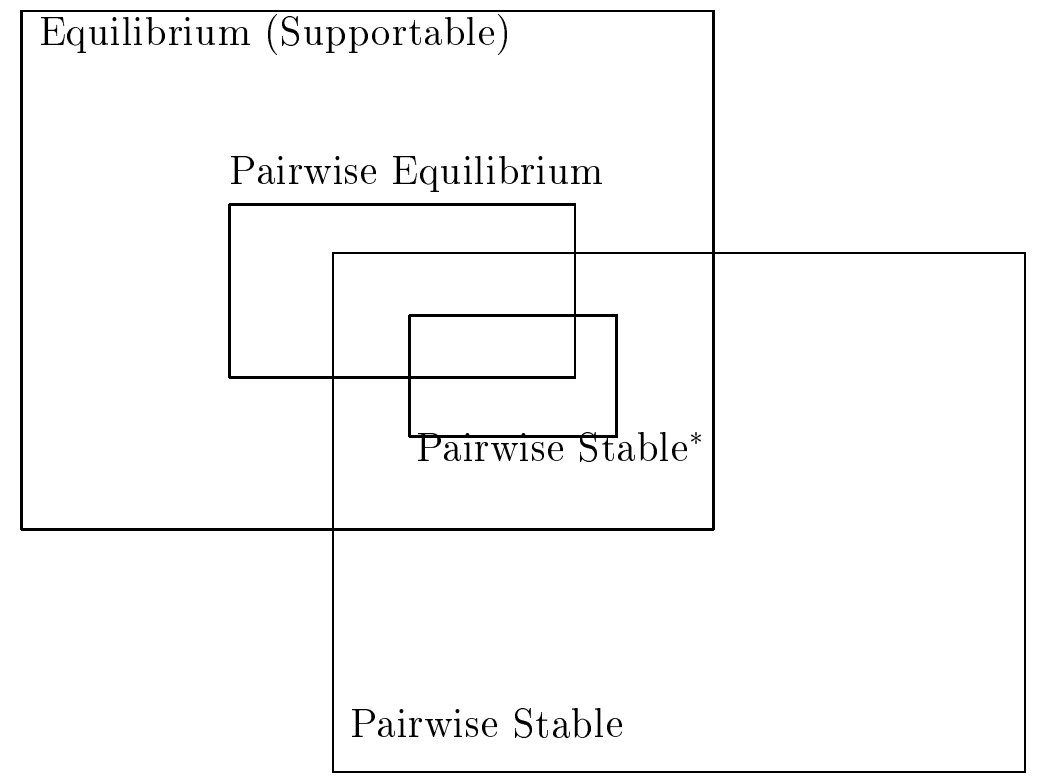

\section{Proposition 24}

(i) The set of pairwise equilibria is a subset of the set of equilibria. 
(ii) If a network $g$ is pairwise stable* relative to a profile of utility functions $u$, then it is supportable via the direct transfer game and it is pairwise stable.

(iii) There exist $u$ and $g$ for which $g$ is pairwise stable* (and thus pairwise stable and supportable), but not supportable via pairwise equilibrium.

(iv) There exist $u$ and $g$ for which $g$ is supported via pairwise equilibrium (and thus supportable) and pairwise stable but not pairwise stable*.

(v) There are networks that are supportable and not pairwise stable nor supportable via pairwise equilibrium.

(vi) There are networks that are pairwise stable and not supportable (nor supportable via pairwise equilibrium, nor pairwise stable*).

(vii) There are networks that are both supportable and pairwise stable, but not pairwise stable* nor supportable via pairwise equilibrium.

(viii) There are networks that are supportable via pairwise equilibrium and not pairwise stable.

(ix) There exist networks that are pairwise stable* (and thus pairwise stable) and at the same time supported via pairwise equilibrium (and thus supportable).

Proof of Proposition 24: (i) follows from the definition of pairwise equilibrium. The pairwise stable part of (ii) is direct. To see the other part of (ii), set $t_{i j}^{i}=t_{i j}^{j}=0$ for each $i j \in g$, and $t_{i j}^{i}=-X$ for each $i j \notin g$, for some $X>0$. For large enough $X$ this forms an equilibrium. To see (iii), consider the empty network in Example 26. To see (iv), see Example 27. To see (v), consider the empty network in Example 1. To see (vi), see Example 25. To see (vii), see Example 28. To see (viii), see Example 26. To see (ix), see the complete network in Example 1.

The examples illustrating the claims in Proposition 24 are as follows.

Example 25 Pairwise stable but not Supportable. 

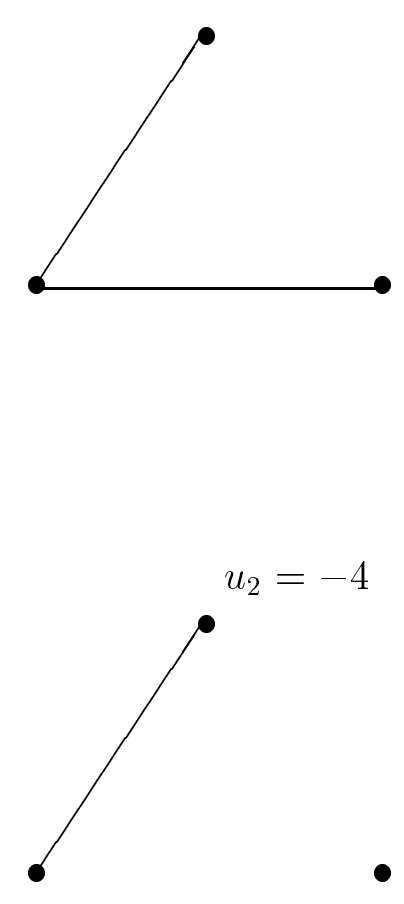

$u_{1}=1$
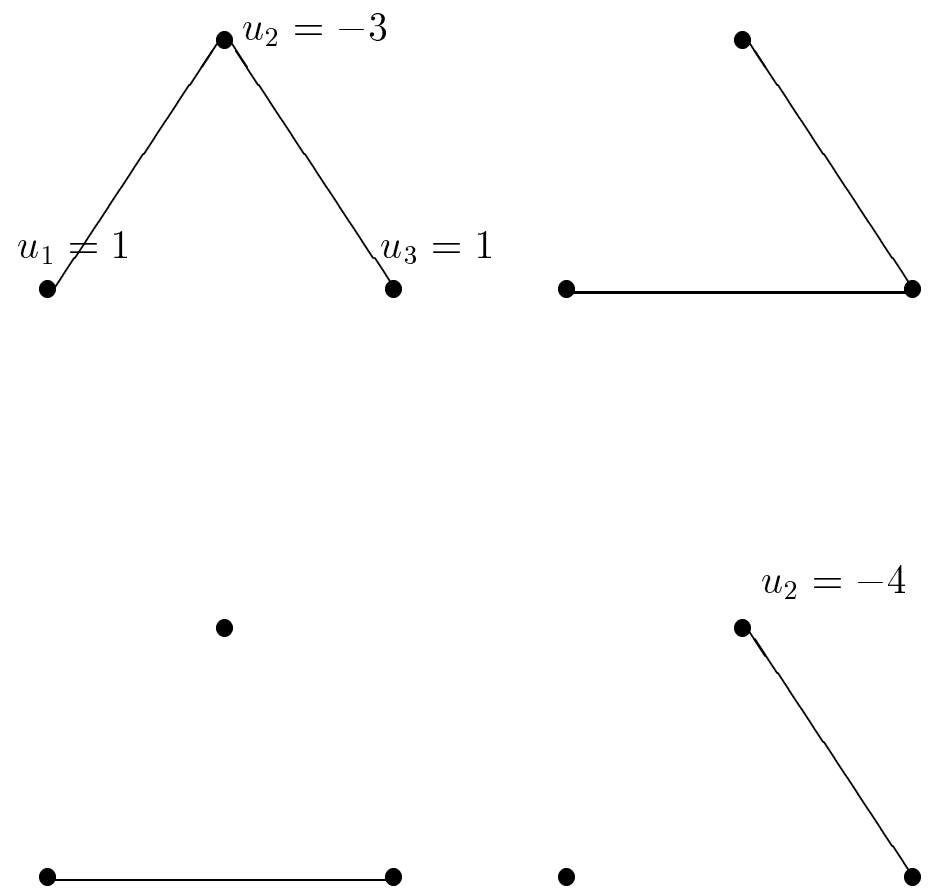

Example 26 Supportable via Pairwise Equilibrium but not Pairwise Stable

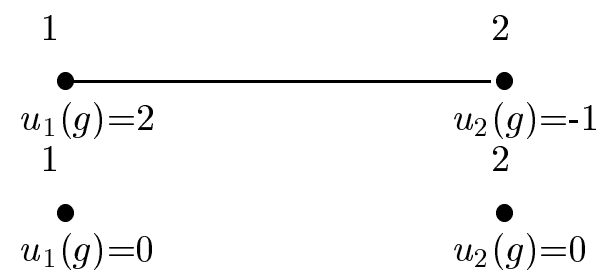

Example 27 Supportable via Pairwise Equilibrium and Pairwise Stable but not Pairwise Stable* 

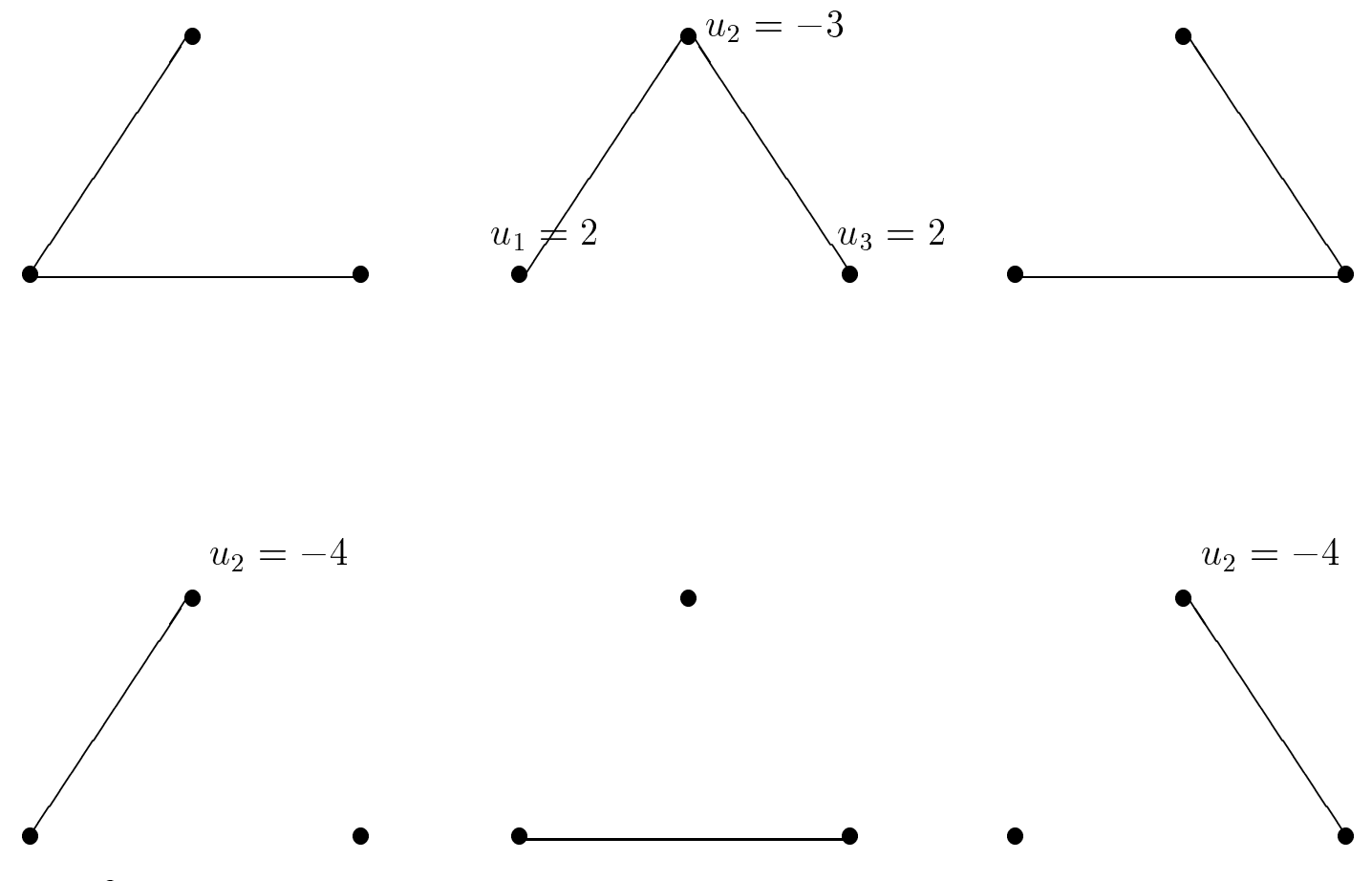

$u_{1}=2$

All other networks have value of 0 . The network $\{12,23\}$ is supportable via pairwise equilibrium and pairwise stable but not pairwise stable*.

Example 28 Supportable and Pairwise Stable but not Pairwise Stable* nor Supportable via Pairwise Equilibrium

This is the same as Example 27, except that the complete network leads to $u_{1}=6$, $u_{2}=-3$, and $u_{3}=-1$. The network $\{12,23\}$ is still supportable and pairwise stable, but no longer supportable via pairwise equilibrium.

\section{Appendix B Proofs}

This Appendix contains the proof of the Propositions in the body of the paper.

Proof of Proposition 5: The network $g$ is supported via an equilibrium of the direct transfer network formation game relative to the profile of utility functions $u$ if and only if there exists a vector of transfers $t$ such that:

- $\sum_{i j \in \ell} t_{i j}^{i} \leq m u_{i}(\ell)$, for all players $i$ and subsets of their links $\ell \subset L_{i}(g)$, and 
- $t_{i j}^{i}+t_{i j}^{j} \geq 0$ for all $i j \in g$.

Furthermore, we know that in equilibrium, we cannot have $t_{i j}^{i}+t_{i j}^{j}>0$ for any $i j$, as then either one of the players would strictly benefit by lowering their $t_{i j}^{i}{ }^{21}$

Therefore, to check whether $g$ is supportable, we can solve the problem

$\min _{t} \sum_{i j \in g} t_{i j}^{i}+t_{i j}^{j}$

subject to:

$-\sum_{i k \in \ell} t_{i k}^{i} \geq-m u_{i}(\ell), \forall i \in N, \ell \subset L_{i}(g)$ and

$t_{i j}^{i}+t_{i j}^{j} \geq 0 \forall i j \in g$

and verify that the solution satisfies:

$\min \sum t_{i j}^{i}+t_{i j}^{j}=0$.

The dual of this problem is ${ }^{22}$

$\max _{\left\{\mu_{\ell}^{i}\right\}_{i \in N, \ell \subset L_{i}(g)},\left\{\nu_{i j}\right\}_{i j \in g}}-\sum_{i} \sum_{\ell \subset L_{i}} \mu_{\ell}^{i} m u_{i}(g, \ell)$ subject to

$\sum_{\ell \subset L_{i}(g): i j \in \ell} \mu_{\ell}^{i}-\nu_{i j}=-1$, for all ordered pairs $i \in N$ and $i j \in g$, and

$\mu_{\ell}^{i} \geq 0$ for all $i \in N$ and $\ell \subset L_{i}(g), \nu_{i j} \geq 0$ for all $i j \in g$.

Since we are free to choose any the $\nu_{i j}$ 's do not appear in the objective function, this problem is equivalent to

$\max _{\left\{\mu_{\ell}^{i}\right\}_{i \in N, \ell \subset L_{i}(g)},\left\{\nu_{i j}\right\}_{i j \in g}}-\sum_{i} \sum_{\ell \subset L_{i}} \mu_{\ell}^{i} m u_{i}(g, \ell)$ subject to

$\sum_{\ell \subset L_{i}(g): i j \in \ell} \mu_{\ell}^{i}-\nu_{i j}=\sum_{\ell \subset L_{j}(g): i j \in \ell} \mu_{\ell}^{j}-\nu_{i j}$ for all ordered pairs $i \in N$ and $i j \in g$, and $\mu_{\ell}^{i} \geq 0$ for all $i \in N$ and $\ell \subset L_{i}(g)$.

As the objective can be set to 0 by setting all of the $\mu_{\ell}^{i}$ 's to 0 , we need only verify that $\sum_{i} \sum_{\ell \subset L_{i}} \mu_{\ell}^{i} m u_{i}(g, \ell)$ is at least 0 for all sets of $\mu_{\ell}^{i}$, s that satisfy the constraints. The constraints correspond to the definition of balanced weights, and thus the proposition follows.

\footnotetext{
${ }^{21}$ We can set $t_{i j}^{i}=t_{i j}^{j}=-X$ for some large enough scalar $X$ for any $i j \notin g$, to complete the specification of the equilibrium strategies.

${ }^{22}$ By standard techniques, one can write the $t_{i j}^{i}=t_{i j}^{i+}-t_{i j}^{i-}$, where $t_{i j}^{i+}$ and $t_{i j}^{i-}$ are both nonnegative. Working across the two inequalities generated by each one of these, we find the equality to -1 .
} 
Proof of Proposition 6: Given Propositions 24 and 5, the first statement follows directly. Thus, the result follows from Lemma 7.

Proof of Lemma 7: Consider $t$ supporting $g$ in either game. In the indirect transfer game, for any $i j \notin g$ and $k \notin i j$, without loss of generality rearrange transfers so that $t_{i j}^{k}=0$. Since $g$ is efficient, and satisfies nonnegative externalities, it must be that $u_{i}(g+i j)+u_{j}(g+i j) \leq u_{i}(g)+u_{j}(g)$, and so $m u_{i}(g, i j)+m u_{j}(g, i j) \leq 0$. Given that $t_{i j}^{k}=0$ for all $k \notin i j$, it follows that any joint deviation by $i$ and $j$ on $i j$ that leads to an improvement for one player, must lead to a loss for the other player.

Proof of Proposition 8: Let $g$ be an efficient graph, then for all link $i j$ we must have

$$
\sum_{k} m u_{k}(g, i j) \geq 0
$$

As the game has nonpositive externalities, this implies that for all links $m u_{k}(g, i j) \leq 0$ for all $k \neq i, j$. Hence, $m u_{i}(g, i j)+m u_{j}(g, i j) \geq 0$. Now by convexity in own-links, $m u_{i}(g, \ell) \geq \sum_{i j \in \ell} m u_{i}(g, i j)$ for any $\ell \subset L_{i}(g)$. Hence

$$
\begin{aligned}
\sum_{i} \sum_{\ell \subset L_{i}(g)} \mu_{\ell}^{i} m u_{i}(g, \ell) & \geq \sum_{i} \sum_{\ell \subset L_{i}(g)} \mu_{\ell}^{i} \sum_{i j \in \ell} m u_{i}(g, i j) \\
& =\sum_{i} \sum_{i j \in g} m u_{i}(g, i j) \sum_{\ell \subset L_{i}(g): i j \in \ell} \mu_{\ell}^{i} \\
& =\sum_{i j \in g}\left(m u_{i}(g, i j) \sum_{\ell \subset L_{i}(g): i j \in \ell} \mu_{\ell}^{i}+m u_{j}(g, i j) \sum_{\ell^{\prime} \subset L_{j}(g): i j \in \ell^{\prime}} \mu_{\ell^{\prime}}^{j}\right)
\end{aligned}
$$

Now, by balancedness, $\sum_{\ell \subset L_{i}(g): i j \in \ell} \mu_{\ell}^{i}=\sum_{\ell^{\prime} \subset L_{j}(g): i j \in \ell^{\prime}} \mu_{\ell^{\prime}}^{j}=\nu_{i j} \geq 0$. Hence,

$$
\sum_{i} \sum_{\ell \subset L_{i}(g)} \mu_{\ell}^{i} m u_{i}(g, \ell) \geq \sum_{i j \in g} \nu_{i j}\left(m u_{i}(g, i j)+m u_{j}(g, i j)\right) \geq 0,
$$

which is the required balance condition.

The Second statement obtains from Lemma 7.

Proof of Proposition 10:(i) Given that $f(2)<f(1)-c$, any two players who are not directly connected will improve their utilities, and thus the total value, by forming a link.

(ii) and (iii). Consider $g^{\prime}$, a component of $g$ containing $m$ players. Let $k \geq m-1$ be the number of links in this component. The value of these direct links is $k(2 f(1)-2 c)$. This leaves at most $m(m-1) / 2-k$ indirect links. The value of each indirect link is at most $2 f(2)$. Therefore, the overall value of the component is at most

$$
k(2 f(1)-2 c)+(m(m-1)-2 k) f(2) .
$$

If this component is a star then its value would be

$$
(m-1)(2 f(1)-2 c)+(m-1)(m-2) f(2) .
$$


Notice that

$$
(1)-(2)=(k-(m-1))(2 f(1)-2 c-2 f(2)),
$$

, which is at most 0 since $k \geq m-1$ and $c>f(1)-f(2)$, and less than 0 if $k>m-1$. The value of this component can equal the value of the star only when $k=m-1$. Any network with $k=m-1$, which is not a star, must have an indirect connection which has a path longer than 2 , getting value at most $2 f(2)$. Therefore, the value of the indirect links will be below $(m-1)(m-2) f(2)$, which is what we get with star.

We have shown that if $c>f(1)-f(2)$, then any component of a efficient network must be a star. Note that any component of a efficient network must have nonnegative value. In that case, a direct calculation using (2) shows that a single star of $m+m^{\prime}$ individuals is greater in value than separate stars of $m$ and $m^{\prime}$ players. Thus if the efficient graph is nonempty, it must consist of a single star. Again, it follows from (2) that if a star of $n$ players has nonnegative value, then a star of $n+1$ players has higher value. Finally, to complete (ii) and (iii) notice that a star encompassing everyone has positive value only when $f(1)+\frac{(n-2)}{2} f(2)>c$.

Proof of Proposition 13: Let $g$ be an efficient network. If $i j \notin g$, let the transfers be $t_{i j}^{i}=t_{i j}^{j}=-X$ and $t_{i j}^{k}=0$ for $k \notin i j$, where $X$ is sufficiently large to be exceed the largest marginal utility of any agent for any set of links. If $i j \in g$, by efficiency $\sum_{k} m u_{k}(g, i j) \geq 0$. If $m u_{k}(g, i j) \geq 0$ for all $k$ set all the transfers $t_{i j}^{k}=0$. If $m u_{i}(g, i j)<0$ and/or $m u_{j}(g, i j)<0$ then set the corresponding $t_{i j}^{i}$ and or $t_{i j}^{j}$ equal to the marginal utility, and then for each $k$ such that $m u_{k}(g, i j)>0$ set $t_{i j}^{k} \in\left[0, m u_{k}(g, i j)\right]$ so that $\sum_{l} t_{i j}^{i}=0$. This is possible by the efficiency of $g$.

These $t$ are such that for any $i j \in g, m u^{l}(g, i j) \geq t_{i j}^{l}$ whenever $l \in i j$ or $l \notin i j$ and $t_{i j}^{l}>0$. Let us argue that this forms an equilibrium of the indirect transfer game.

First, note that by the definition of $X$, if there exists an improving deviation, there will exist one that only changes $t$ 's on links in $g$.

By convexity in all links, if there exists a deviation that is improving for some $l$ on $t^{l}$ on some set of links, then there exists some deviation that involves at most one link $t_{i j}^{l}$, with the possibility that $l \in i j$. For $i j \in g$, increasing transfers is costly and does not change the outcome. Reducing transfers implies that the link will not be formed. Such a deviation cannot be profitable as $m u^{l}(g, i j)-t_{i j}^{l} \geq 0$ if $l \in i j$ or if $l \notin i j$ and $t_{i j}^{l}>0$. It is not possible to lower $t_{i j}^{l}$ below 0 if $l \notin i j$.

The last claim in the Proposition follows from Lemma 7.

Proof of Corollary 14 We first show that the efficient network is supported in a strong pairwise equilibrium. Clearly, an efficient network must satisfy ij $\in g$ if and only if $\sum_{k} w_{i j}^{k} \geq 0$. Consider then the following transfer scheme. For any link such that $\sum_{k} w_{i j}^{k} \geq 0$. If $w_{i j}^{i} \geq 0$ and $w_{i j}^{j} \geq 0$, let $t_{i j}^{k}=0$ for all $k$. If at least one of the two involved 
players has a negative marginal utility from that link, consider all players $k$ for which $w_{i j}^{k}>0$ and set transfers so that $t_{i j}^{k}=w_{i j}^{k}-\left(\sum_{k} w_{i j}^{k} /|K|\right)$ and for $i$ such that $w_{i j}^{i}<0$ set $t_{i j}^{i}=w_{i j}^{i}$. For any link such that $\sum_{k} w_{i j}^{k}<0$ set transfers $t_{i j}^{k}=X$ where $X$ is very large. For any $i j \in g(t)$, it is clear that those transfers form an equilibrium strategy. If $i j \notin g(t)$, there cannot be any transfer scheme such that $\sum_{k} t_{i j}^{k} \geq 0$ and $\sum_{k} w_{i j}^{k}-\sum_{k} t_{i j}^{k}>0$.

Next, suppose by contradiction that an inefficient network is supported in a strong pairwise equilibrium. As $g$ is inefficient, there must exist either $i j \in g$ and $\sum_{k} w_{i j}^{k}<0$ or $i j \notin g$ and $\sum_{k} w_{i j}^{k}>0$. Because payoffs satisfy nonnegative externalities, if $\sum_{k} w_{i j}^{k}<0$ then $w_{i j}^{i}+w_{i j}^{j}<0$. Hence, one of the players must have a profitable deviation by changing transfers so as to sever the link. If $\sum_{k} w_{i j}^{k}>0$, construct a transfer scheme as above. ( If $w_{i j}^{i} \geq 0$ and $w_{i j}^{j} \geq 0$, let $t_{i j}^{k}=0$ for all $k$. If at least one of the two involved players has a negative marginal utility from that link, consider all players $k$ for which $w_{i j}^{k}>0$ and set transfers so that $t_{i j}^{k}=w_{i j}^{k}-\left(\sum_{k} w_{i j}^{k} /|K|\right)$ and for $i$ such that $w_{i j}^{i}<0$ set $t_{i j}^{i}=w_{i j}^{i}$. Under this transfer scheme the link is formed and all players increase their utilities.

Proof of Proposition 15: The necessity of $\sum_{i \in N\left(g^{\prime}\right)} u_{i}(g)=\sum_{i \in N\left(g^{\prime}\right)} y_{i}$ for all $g^{\prime} \in C(g)$, and $y_{i} \neq u_{i}(g)$ implies $i \in N(g)$ follow from the balance of transfers across components and the observation that in equilibrium the transfers will sum to 0 on any link that is formed.

To complete the proof, let us show that any such network $g$ and allocation $y$ can be supported as an equilibrium.

Let $Y=3 \max \left\{\max _{i}\left|y_{i}\right| ; \max _{i, g^{\prime}}\left|u_{i}\left(g^{\prime}\right)\right|\right\}$.

For $g^{\prime} \neq g$, set $t_{i j}^{i}\left(g^{\prime}\right)=-Y$ for all $i$ and $j$.

For $g$, set transfers as follows. For any $i j \notin g$ set $t_{i j}^{i}=t_{i j}^{j}=-Y$.

For $i j \in g$ we set transfers as follows.

Consider a component $g^{\prime} \in C(g)$.

Find a tree $h \subset g^{\prime}$ such that $N(h)=N\left(g^{\prime}\right) .{ }^{23}$

Let player $i$ be a root of the tree. ${ }^{24}$ Consider each $j$ who has just one link in the tree. There is a unique path from $j$ to $i$. Let this path be the network $h^{\prime}=\left\{i_{1} i_{2}, \ldots, i_{K-1} i_{K}\right\}$, where $j=i_{1}$ and $i=i_{K}$.

\footnotetext{
${ }^{23} \mathrm{~A}$ tree is a network that consists of a single component and has no cycles (paths such that every player with a link in the path has two links in the path).

${ }^{24} \mathrm{~A}$ root of the tree is a player who lies on any path that connects any two players who each have just one link in the tree.
} 
Iteratively, for each $k \in\{1, \ldots, K\} \operatorname{set}^{25}$

$$
t_{i_{k-1} i_{k}}^{i_{k}}=\sum_{k^{\prime}<k} y_{i_{k^{\prime}}}-u_{i_{k^{\prime}}}(g)
$$

and

$$
t_{i_{k} i_{k+1}}^{i_{k}}=\sum_{k^{\prime} \leq k}-\left(y_{i_{k^{\prime}}}-u_{i_{k^{\prime}}}(g)\right)
$$

Do this for each path in the tree.

For any link $i j \in g$ but $i j \notin h$, set $t_{i j}^{i}=t_{i j}^{j}=0$.

Under these transfers, $g$ will be the network that forms and $y$ will be the payoff vector. Let us check that there are no improving deviations.

Consider a deviation that leads to another network $g^{\prime} \neq \emptyset$ being formed. This must involve a net loss for any $i$ as $i$ 's payoff must be below $u_{i}\left(g^{\prime}\right)-Y$. Next, consider a deviation that leads to the empty network. It must be that that the deviating player is $i \in N(g)$ in which case the new payoff is 0 for $i$, which cannot be improving as $y_{i} \geq 0$. So, consider a deviation by a player $i$ that still leads to $g$ being formed. Player $i$ 's promises $t_{i j}^{i}(g)$ can only have increased, which can only lower $i$ 's payoff.

\section{Proof of Proposition 17:}

Let $Y=3 \max \left\{\max _{i}\left|y_{i}\right| ; \max _{i, g^{\prime}}\left|u_{i}\left(g^{\prime}\right)\right|\right\}$.

For $g^{\prime} \neq g$, set $t_{i j}^{i}\left(g^{\prime}\right)=-Y$ for all $i$ and $j$, and set $t_{j k}^{i}\left(g^{\prime}\right)=0$ for $i \notin j k$.

For $g$, set transfers as follows. Let $A=\left\{i \mid y_{i}>u_{i}(g)\right\}$ and $B=\left\{i \mid y_{i}<u_{i}(g)\right\}$.

For $i \in A$ let $\ell_{i}(g)$ be the number of links that $i$ has in $g$. Set $t_{i j}^{i}(g)=\frac{-y_{i}+u_{i}(g)}{\ell_{i}(g)}$ if $i j \in g$ and set $t_{i j}^{i}(g)=-Y$ if $i j \notin g$, and $t_{j k}^{i}=0$ otherwise.

For $i \in B$ let

$$
\lambda_{i}=\frac{u_{i}(g)-y_{i}}{\sum_{j \in B} u_{j}(g)-y_{j}} .
$$

Then for $i \in B$ set

$$
\begin{gathered}
t_{j k}^{i}(g) \\
=\lambda_{i}\left(\frac{y_{j}-u_{j}(g)}{\ell_{j}(g)}+\frac{y_{k}-u_{k}(g)}{\ell_{k}(g)}\right) \text { if } j k \in g, j \in A \text { and } k \in A,
\end{gathered}
$$

\footnotetext{
${ }^{25}$ For $k=1$ only the second equation applies, and for $k=K$ only the first applies.
} 


$$
\begin{gathered}
=\lambda_{i}\left(\frac{y_{j}-u_{j}(g)}{\ell_{j}(g)}\right) \text { if } j k \in g, j \in A \text { and } k \notin A, \\
=-Y \text { if } j k \notin g \text { and } i \in j k \text {, and } \\
=0 \text { otherwise. }
\end{gathered}
$$

For $i \notin A \cup B$, set $t_{i j}^{i}=-Y$ if $i j \notin g$ and $t_{j k}^{i}=0$, otherwise.

Under these transfers, $g$ will be the network that forms and $y$ will be the payoff vector. Let us check that there are no improving deviations.

Consider a deviation that leads to another network $g^{\prime} \neq \emptyset$ being formed. This must involve a net loss for any $i$ as $i$ 's payoff must be below $u_{i}\left(g^{\prime}\right)-Y$. Next, we consider a deviation by a player $i$ that leads to the empty network. This cannot be improving as $y_{i} \geq 0$. So, consider a deviation by a player $i$ that still leads to $g$ being formed. Player $i$ 's promises $t_{j k}^{i}(g)$ can only have increased, which can only lower $i$ 's payoff. I

Proof of Proposition 23: It is clear that the set of pairwise equilibria is a subset of the set of equilibria of the direct transfer game. Let us show that any network supportable as a pairwise equilibrium is also pairwise stable with transfers. Consider a pairwise equilibrium $\widehat{t}$. For any link $i j \in g$, player $i$ prefers to announce $\widehat{t_{i j}^{i}}$ than any transfer $X$ such that $X+\widehat{t_{i j}^{j}}<0$. Hence, $u_{i}(g)-\widehat{t_{i j}^{i}} \geq u_{i}(g-i j)$. Similarly, $u_{j}(g)-\widehat{t_{i j}^{j}} \geq u_{i}(g-i j)$. Summing up the two inequalities, $u_{i}(g)+u_{j}(g)-\left(\widehat{t_{i j}^{i}}+\widehat{t_{i j}^{j}}\right) \geq u_{i}(g-i j)+u_{j}(g-i j)$ and as $\left(\widehat{t_{i j}^{i}}+\widehat{t_{i j}^{j}}\right) \geq 0, u_{i}(g)+u_{j}(g) \geq u_{i}(g-i j)+u_{j}(g-i j)$. Conversely, suppose that $i j \notin g$. If $u_{i}(g)+u_{j}(g)>u_{i}(g-i j)+u_{j}(g-i j)$, define a new transfer vector $\widetilde{t}$ where $\widehat{t}_{k l}^{h}=\widehat{t_{k l}^{h}}$ for all $k l \neq i j$ and $\widetilde{t}_{i j}^{i}=u_{i}(g)-u_{i}(g-i j)-\varepsilon, \widetilde{t}_{i j}^{j}=u_{j}(g)-u_{j}(g-i j)-\varepsilon$ where $\varepsilon$ is chosen so that $\widetilde{t}_{i j}^{i}+\widetilde{t}_{i j}^{j} \geq 0$. It follows that $u_{i}(g(\widetilde{t}))-\sum_{k, i k \in g(\widetilde{t})} \widetilde{t}_{i k}^{i}=u_{i}(g-i j)-\sum_{k \neq j, i k \in g(\widetilde{t})} \widehat{t_{i k}^{i}}+\varepsilon>$ $u_{i}(g(\widehat{t}))-\sum_{k, i k \in g(\widehat{t})} \widehat{t_{i k}^{i}}$ and similarly, $u_{j}(g(\widetilde{t}))-\sum_{k, j k \in g(\widetilde{t})} \widetilde{t}_{j k}^{j}>u_{j}(g(\widehat{t}))-\sum_{k, j k \in g(\widehat{t})} \widehat{t_{j k}^{j}}$, contradicting the definition of pairwise equilibrium.

Finally, let us argue that any network $g$ that is supportable and is also pairwise stable with transfers is supportable as a pairwise equilibrium. Consider an equilibrium $\hat{t}$ that supports $g$. We argue that $\widehat{t}$ must also be a pairwise equilibrium. Suppose to the contrary that there exists some $i j \notin g$ such that

$$
u_{i}(g+i j)-\sum_{i k \in g} t_{i k}^{i}-\widehat{t}_{i j}^{i} \geq u_{i}(g)-\sum_{i k \in g} t_{i k}^{i}
$$

and

$$
u_{j}(g+i j)-\sum_{j k \in g} t_{j k}^{j}-\widehat{t}_{i j}^{j} \geq u_{j}(g)-\sum_{j k \in g} t_{j k}^{j},
$$

with one inequality holding strictly, and where $\widehat{t}_{i j}^{i}+\widehat{t}_{i j}^{j} \geq 0$ (as otherwise the link $i j$ does not form and the payoffs could not have changed). Thus,

$$
u_{i}(g+i j)-\widehat{t}_{i j}^{i}+u_{j}(g+i j)-\widehat{t}_{i j}^{j}>u_{i}(g)+u_{j}(g) .
$$


Since $\widehat{t}_{i j}^{i}+\widehat{t}_{i j}^{j} \geq 0$ it follows that

$$
u_{i}(g+i j)+u_{j}(g+i j)>u_{i}(g)+u_{j}(g),
$$

which contradicts the fact that $g$ is pairwise stable with transfers.

Proof of Proposition 22: For $n=2$, the Proposition is straightforward, as the only networks are the empty and single link network. The single link network is supportable as a (strong pairwise) equilibrium if and only if it has nonnegative value. In the case where a link's value is nonpositive, the empty network is clearly supportable as a (strong pairwise) equilibrium.

So consider a setting where $n \geq 3$. Let $g$ be such that $\sum_{i}(g) \geq 0$.

Let $Y=3 \max _{i, g^{\prime}}\left|u_{i}\left(g^{\prime}\right)\right|$.

For $g^{\prime} \neq g$, set $t_{i j}^{i+}\left(g^{\prime}\right)=t_{i j}^{i-}\left(g^{\prime}\right)=-Y$ for all $i$ and $j$, and set $t_{j k}^{i}\left(g^{\prime}\right)=0$ for $i \notin j k$. Set $m_{j}^{i}\left(g^{\prime}\right)=+$ if $i j \notin g^{\prime}$ and $m_{j}^{i}\left(g^{\prime}\right)=-$ if $i j \in g^{\prime}$. Note that under these rules, $g\left(t\left(g^{\prime}\right), m\left(g^{\prime}\right)\right)$ (the links that would form given these announcements) is the complement of $g^{\prime}$.

For $g$, set transfers as follows. Let $\bar{u}=\frac{\sum_{i} u_{i}(g)}{n}$ be the average payoff from $g$, which is at least 0 . Let $A=\left\{i \mid u_{i}(g) \geq \bar{u}\right\}$ and $B=\left\{i \mid u_{i}(g)<\bar{u}\right\}$, and $n_{A}$ and $n_{B}$ be the corresponding cardinalities.

Set $m_{j}^{i}\left(g^{\prime}\right)=+$ for all $i j \in g$ and $m_{j}^{i}\left(g^{\prime}\right)=-$ if $i j \notin g$. Set the $t$ 's as follows. If $n_{B}=0$, then set $t_{i j}^{k}=0$ for all $k$ and $i j$.

For $n_{B}>0$, let $\lambda_{j}=\frac{\bar{u}-u_{j}(g)}{\sum_{k \in B} \bar{u}-u_{k}(g)}$ for $k \in B$ and $\lambda_{j}=0$ if $j \in A$.

For $i \in B$ set $t_{i j}^{i+}(g)=t_{i j}^{i-}\left(g^{\prime}\right)=\frac{\bar{u}-u_{i}(g)}{n-1}$ for all $j$, and set $t_{j k}^{i \cdot}(g)=0$ when $i \notin j k$. For $i \in A$ set $t_{i j}^{i+}(g)=t_{i j}^{i-}\left(g^{\prime}\right)=\lambda_{j} \frac{u_{i}(g)-\bar{u}}{n-1}$ for all $j$, and set $t_{j k}^{i \cdot}(g)=\left(\lambda_{j}+\lambda_{k}\right) \frac{u_{i}(g)-\bar{u}}{n-1}$ when $i \notin j k$.

Under these announcements, $g$ is formed and each player's payoff is $\bar{u}$. Consider any deviation by a player $i$. Given the announced $t_{-i}$ and $m_{-i}$ (and the fact that there are three or more players), $i$ can only induce the empty network and a payoff of 0 . This can not be improving. Consider a deviation by some group of players $S$ on the announcements pertaining to a link $i j$. Again, they can only induce the empty network and a payoff of 0 , or else the network $g$ and some reallocation of their own payoffs. Neither of these deviations can make each member of the group as well off and some better off. 